\title{
Insights from a Paradigm Shift: How the Poly(A)-Binding Protein Brings Translating mRNAs Full Circle
}

\author{
Daniel R. Gallie \\ Department of Biochemistry, University of California, Riverside, CA 92521-0129, USA \\ Correspondence should be addressed to Daniel R. Gallie; drgallie@citrus.ucr.edu
}

Received 18 May 2014; Accepted 5 July 2014; Published 18 August 2014

Academic Editor: Yuri L. Lyubchenko

Copyright ( 2014 Daniel R. Gallie. This is an open access article distributed under the Creative Commons Attribution License, which permits unrestricted use, distribution, and reproduction in any medium, provided the original work is properly cited.

\begin{abstract}
In recent years, our thinking of how the initiation of protein synthesis occurs has changed dramatically. Initiation was thought to involve only events occurring at or near the $5^{\prime}$-cap structure, which serves as the binding site for the cap-binding complex, a group of translation initiation factors (eIFs) that facilitate the binding of the $40 \mathrm{~S}$ ribosomal subunit to an mRNA. Because the poly(A)-binding protein (PABP) binds the poly(A) tail present at the $3^{\prime}$-terminus of an mRNA, it was long thought to play no role in translation initiation. In this review, I present evidence from my laboratory that has contributed to the paradigm shift in how we think of mRNAs during translation. The depiction of mRNAs as straight molecules in which the poly(A) tail is far from events occurring at the $5^{\prime}$-end has now been replaced by the concept of a circular mRNA where the interaction between PABP and the cap-binding complex bridges the termini of an mRNA and promotes translation initiation. The research from my laboratory supports the new paradigm that translation of most mRNAs requires a functional and physical interaction between the termini of an mRNA.
\end{abstract}

\section{Introduction}

Our understanding of how mRNAs are translated into proteins has undergone a paradigm shift in recent years. Prior to this shift, a translating mRNA undergoing translation was thought of as a straight molecule and protein synthesis was considered a linear process that encompassed three phases. In this prior view, the first phase, that is, translation initiation, begins with events that only involve the $5^{\prime}$-end of an mRNA in which the subunits of a ribosome are recruited and assembled at the initiation codon and conclude with the synthesis of the first peptide bond (Figure 1). This is followed by the elongation phase, that is, the ribosome-catalyzed decoding of the open reading frame into protein and finally the recognition of the stop codon and release of the nascent peptide from the ribosome. In this paper, I will illustrate how our understanding of how mRNAs undergoing translation in eukaryotes fundamentally changed from a view in which translation initiation is orchestrated by events only involving the $5^{\prime}$-end of an mRNA to one in which the mRNA is circularized by functional and physical interactions between the termini of an mRNA. As my research involves translation in plants, findings from this kingdom this will be emphasized. However, the similarities and differences in the interactions of the machinery involved in ribosome recruitment in plants and those of other eukaryotes will be also be discussed.

\section{The Discovery of Synergy}

The basic machinery and reactions involved in protein synthesis, that is, the ribosome, charged tRNAs, and mRNAs, are conserved throughout all kingdoms. It is the way in which the protein synthetic machinery is assembled during translation initiation that differs between eukaryotes and bacteria, principally in how the small subunit of the ribosome is recruited to an mRNA. In bacteria, recruitment involves base pairing between a short sequence at the $3^{\prime}$-end of the $16 \mathrm{~S}$ rRNA in the $30 \mathrm{~S}$ ribosomal subunit and the Shine-Dalgarno sequence in the mRNA [1]. The base pairing between these RNAs positions the $30 \mathrm{~S}$ ribosomal subunit such that the first AUG (which serves as the initiation codon for most coding regions) within 5 to 10 nucleotides downstream of the Shine-Dalgarno sequence is recognized as the initiation codon. Although three translation factors are involved in 


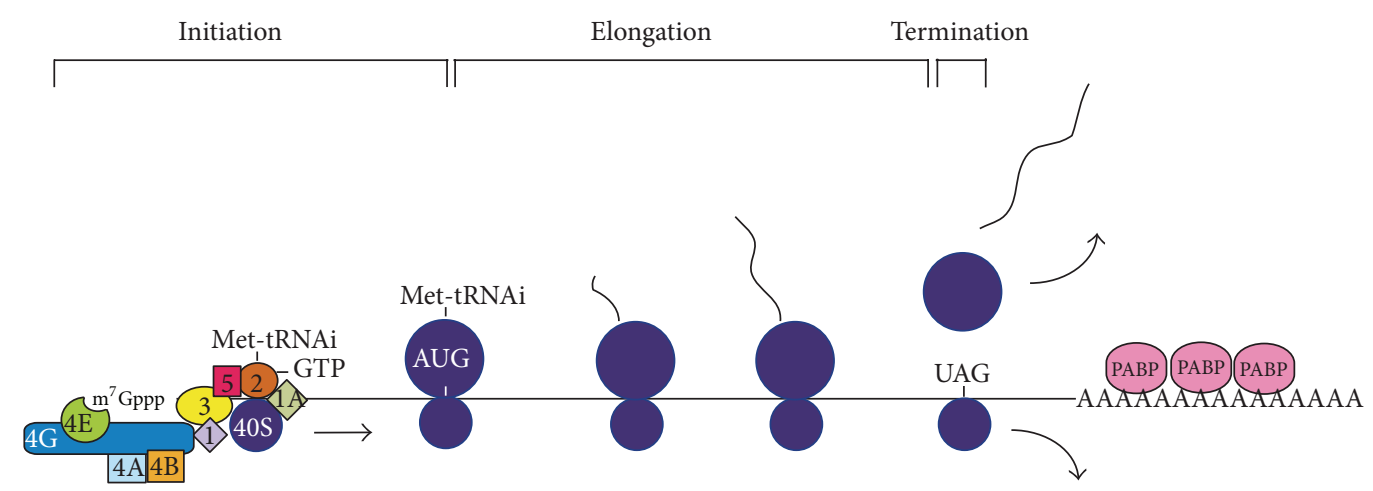

FIGURE 1: Overview of the three steps during protein synthesis in eukaryotes. During translation initiation, a $40 \mathrm{~S}$ subunit is recruited at the $5^{\prime}$-cap through the interaction between eIF3 and eIF4G, the large subunit of the eIF4F complex that is bound to the $5^{\prime}$-cap through the eIF4E subunit. Secondary structure in the $5^{\prime}$-UTR is removed through the ATP-dependent helicase activity of eIF4A which is assisted by eIF4B. The $40 \mathrm{~S}$ subunit then scans down the $5^{\prime}$-UTR to locate the first AUG in a good context at which point the $60 \mathrm{~S}$ subunit joins. Protein synthesis begins with the formation of the first peptide bond at which point the initiation phase ends and the elongation phase begins until the $80 \mathrm{~S}$ ribosome reaches a termination codon at which point translation terminates releasing the nascent peptide. Not all initiation factors involved in translation initiation are shown and the role of PABP in translation initiation is not depicted.

promoting the initiation of protein synthesis in bacteria, they function principally to maintain the dissociation of the $30 \mathrm{~S}$ and $50 \mathrm{~S}$ subunits of nontranslating ribosomes and to recruit a tRNA ${ }^{\text {fMet }}$ to the $30 \mathrm{~S}$ ribosomal subunit.

In contrast, the eukaryotic $40 \mathrm{~S}$ ribosomal subunit is not recruited to an mRNA through base pairing as the eukaryotic $18 \mathrm{~S}$ rRNA lacks the region corresponding to the Shine-Dalgarno sequence of the bacterial $16 \mathrm{~S}$ rRNA. The identification of the initiation codon in most eukaryotic mRNAs involves a scanning mechanism in which a $40 \mathrm{~S}$ ribosomal subunit is recruited at or near the $5^{\prime}$-cap structure $\left(\mathrm{m}^{7} \mathrm{GpppN}\right)$ of an mRNA and scans the $5^{\prime}$-untranslated region $\left(5^{\prime}\right.$-UTR) in a $5^{\prime}$ to $3^{\prime}$ direction in search of the first AUG in a good context at which point a $60 \mathrm{~S}$ subunit joins to form the translationally competent $80 \mathrm{~S}$ ribosome (Figure 2) [2]. The context of an initiation codon is defined by the sequence immediately surrounding an AUG, principally by the -3 and +4 nucleotides (where the A of an AUG is designated as +1) [2].

Recruitment of a $40 \mathrm{~S}$ ribosomal subunit is facilitated through the action of multiple initiation factors that also aid in the assembly of the $80 \mathrm{~S}$ ribosome at the initiation codon [3-5]. For most mRNAs, $40 \mathrm{~S}$ subunit recruitment requires the action of the eIF4 group of initiation factors that comprise the cap-binding complex which include eIF4F and eIF4B. eIF4F is comprised of eIF4E, which is the subunit responsible for binding the $5^{\prime}$-cap structure; eIF4A, a DEAD/Hbox, ATP-dependent, RNA helicase; and eIF4G, a modular scaffolding protein that interacts with eIF4E, eIF4A, and eIF3 (required for $40 \mathrm{~S}$ binding to an mRNA) [6] (Figure 2). The functions of the cap-binding complex are highly conserved among eukaryotes and are required to promote the assembly of the translation machinery to an mRNA and prepare it for translation. For example, as a DEAD/H-box helicase, eIF4A uses energy from ATP hydrolysis to unwind secondary structure present in the $5^{\prime}$-leader of an mRNA that might otherwise inhibit $40 \mathrm{~S}$ subunit scanning [4]. eIF4B promotes the ATP-dependent, RNA helicase activity of eIF4A and eIF4F [7-17]. eIF4G is the largest subunit of eIF4F and interacts with most of the factors required to prepare an mRNA for $40 \mathrm{~S}$ ribosomal subunit recruitment. eIF3 is the largest of the translation initiation factors that binds to free $40 \mathrm{~S}$ subunits and, through its interaction with eIF4G, serves to facilitate $40 \mathrm{~S}$ subunit binding to an mRNA [3-5].

Because mRNAs were typically depicted as straight linear molecules since the discovery of messenger RNAs, the capbinding complex and its interaction with the $5^{\prime}$-cap were thought to be solely responsible for preparing an mRNA for $40 \mathrm{~S}$ subunit recruitment. Although polyadenylation that follows transcription of mRNAs and the poly(A)-binding protein $(\mathrm{PABP})$ that binds the poly $(\mathrm{A})$ tail were well known, depiction of mRNAs as straight molecules positioned the poly(A) tail far from the events that occur at the $5^{\prime}$-terminus during translation initiation and as such made it seem unlikely that it could participate in the initiation process. From my experience working with tobacco mosaic virus, whose genome is a single strand of RNA encoding the proteins needed to replicate and package new copies of the genome, I knew that RNA was almost never present in a linear form and indeed could even fold into knot-like structures, known as pseudoknots, particularly in the $3^{\prime}$-untranslated region which was the region I found most intriguing.

Although the prevailing notion of PABP's function when bound to a poly $(A)$ tail was to prevent $3^{\prime}$ degradation of an mRNA, the idea that mRNAs are highly flexible suggested to me that PABP may play a role during translation as there was no physical reason preventing it from being in close proximity to the $5^{\prime}$-terminus. Because I had developed a procedure for delivering in vitro transcribed mRNAs into living plant protoplasts [18], I generated four nearly identical luciferase reporter mRNAs that differed only in whether they contained a $5^{\prime}$-cap and/or a poly(A) tail. Once these mRNAs were individually delivered to plant protoplasts, the resulting luciferase activity would serve as a measure of how well 


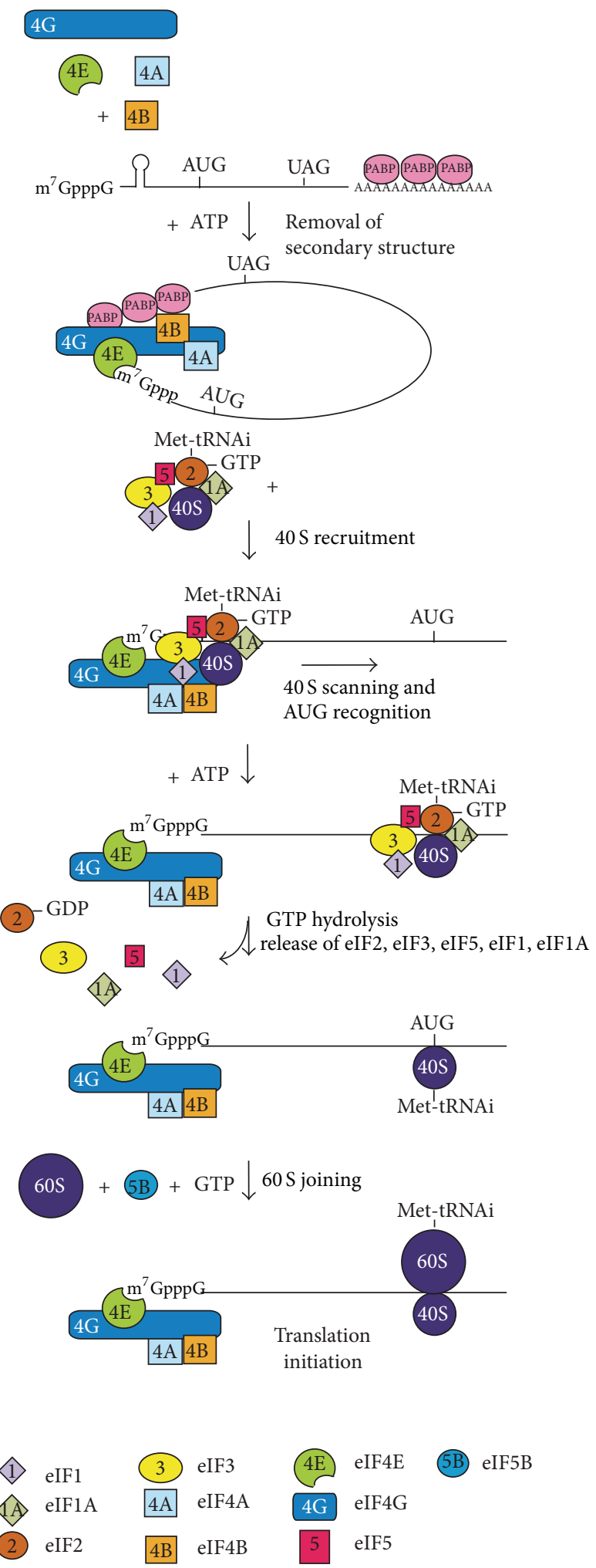

Figure 2: Detailed depiction of the steps during translation initiation. In the first step, eIF4E, eIF4G, eIF4A, and eIF4B are recruited to the $5^{\prime}$-cap of an mRNA. Using the hydrolysis of ATP, eIF4A assisted by eIF4B removes secondary structure in the $5^{\prime}$-UTR. The interactions between PABP and eIF4G as well as eIF4B that circularize the mRNAs are depicted. The $43 \mathrm{~S}$ complex, containing the $40 \mathrm{~S}$ subunit, eIF1, eIF1A, eIF2, eIF3, and eIF5, is recruited to the $5^{\prime}$-cap through the interaction between eIF3 and eIF4G and the $40 \mathrm{~S}$ subunit scans down the $5^{\prime}$-UTR. Whether eIF4F remains bound at the $5^{\prime}$-cap is not known. Upon location of the first AUG in a good context, the initiation factors are released and eIF5B assists the joining of the $60 \mathrm{~S}$ subunit to form the $80 \mathrm{~S}$ ribosome at which point protein synthesis begins. A key to the initiation factors depicted is included in the figure. 
each mRNA was translated. The luciferase reporter mRNAs containing a $5^{\prime}$-cap or a poly(A) tail yielded moderately more luciferase activity than the luciferase mRNA lacking both elements [19]. The presence of a $5^{\prime}$-cap and a poly(A) tail, however, resulted in a synergistic increase in luciferase activity, suggesting that the $5^{\prime}$-cap and poly(A) tail functioned together to promote protein synthesis from the mRNA [19].

But the question was whether the $5^{\prime}$-cap and poly(A) tail synergistically increased the translatability of an mRNA (i.e., the rate at which an mRNA is translated) or whether the $5^{\prime}$ cap and poly(A) tail synergistically increased the stability of an mRNA (which allows an mRNA to be translated for a longer period of time). By measuring each individually, I saw that the $5^{\prime}$-cap and poly (A) tail increased the stability of the mRNA but their greatest synergistic effect was on increasing the translatability of the mRNA. By using similar approaches with mammalian and yeast cells, I observed the same results in animals and yeast [19]. This was my first evidence of the functional cooperation between the termini of an mRNA during translation and, mostly likely, the explanation for the functional cooperation was a physical interaction between the cap-binding complex and PABP (Figure 2), as these were the factors that bound the $5^{\prime}$-cap and poly(A) tail, respectively.

Interestingly, I later observed that many mRNAs naturally lacking a $5^{\prime}$-cap or a poly(A) tail have evolved functional equivalents to these and they also functionally interact [2026]. For example, tobacco mosaic virus genomic RNA, which functions as an mRNA, terminates not in a poly(A) tail but in a $3^{\prime}$-untranslated region composed of RNA pseudoknots as mentioned above. A subgroup of these RNA pseudoknots within the $3^{\prime}$-untranslated region serves as the functional equivalent to a poly(A) tail to enhance translation but this pseudoknot region is entirely dependent on the presence of the cap structure at the $5^{\prime}$-end of the mRNA to do so $[20,21]$. This suggested to me that the interaction between the termini of an mRNA is a common feature among mRNAs and that such an interaction provides a benefit to the synthesis of protein from an mRNA.

\section{When Functional Becomes Physical}

In order to test the hypothesis that the functional cooperation between the $5^{\prime}$-cap and poly(A) tail was facilitated by a physical interaction between the cap-binding complex and PABP, I needed to determine whether PABP could interact with one or more components of the cap-binding complex. Because the genes for PABP and all of the subunits of the capbinding complex were not available for the plant factors at the time which would have allowed us to use recombinant forms of the proteins, we used purified factors isolated from wheat embryos which are rich in these proteins. We observed that PABP interacts with eIF4B and eIF4G (or eIFiso4G, a second isoform found only in plants) [27]. The interaction between PABP and eIF4G agreed with findings in yeast [28]. Initial attempts to determine whether PABP could interact with eIF4G in animals failed until it was discovered that the eIF4G cDNA available to researchers at the time did not contain the entire open reading frame. Once a longer cDNA was isolated, which enabled the production of an eIF4G protein with a longer $\mathrm{N}$-terminal region, the interaction between PABP and eIF4G was finally observed [29-31].

When I was on sabbatical in the laboratory of Virginia Pain and Simon Morley at the University of Sussex in 1999, I suggested to Martin Bushell, a graduate student who was studying mammalian protein synthesis, that he investigate whether PABP in animals could interact with eIF4B. He took the suggestion and discovered, as in plants, that PABP and eIF4B do interact [32]. To date, no interaction between PABP and eIF4B has been reported for yeast. Therefore, the interaction between PABP and eIF4G is conserved through three kingdoms of eukaryotes while the interaction between PABP and eIF4B is conserved among higher eukaryotes at least. This conservation strongly suggested the interactions were important but it also raised several questions. First, what region in each protein was required for the interaction? Second, did PABP interact with eIF4G and eIF4B independently, cooperatively, or were the two interactions mutually exclusive? What were the functional consequences of their interactions on translation? This then became the next area of discovery for us. Our use of plants as a model system allowed us to show that similarities do exist with animals and yeast but we also discovered aspects that are unique to plants.

\section{4. eIF4G: Central to the Interaction between PABP and the Cap-Binding Complex}

eIF4G is the largest subunit of eIF4F and, through its binding to eIF4E, is recruited to the $5^{\prime}$-cap structure (Figure 2). eIF4G has no known catalytic function but serves as a scaffold that, through its interactions with several initiation factors and associated proteins, prepares an mRNA for binding to a 40 $S$ ribosomal subunit and its subsequent scanning. eIF4G also promotes $40 \mathrm{~S}$ subunit recruitment to an mRNA through its binding to eIF3 that in turn binds to free $40 \mathrm{~S}$ subunits [3-5].

eIF4G is notable in that it contains HEAT (Huntington, Elongation Factor 3, PR65/A, TOR) repeat domains (Figure 3), each composed of antiparallel $\alpha$-helical hairpins known as HEAT repeats $[33,34]$. Plant eIF4G contains two HEAT domains, whereas mammalian eIF4G contains three and yeast eIF4G contains just one. The HEAT domain present in all known eIF4G homologs is HEAT-1/MIF4G which lies in the middle region of eIF4G. This HEAT domain in plant, animal, and yeast eIF4G is composed of five HEAT repeats in a helical stack [33]. The HEAT-2/MA3 domain, also composed of five HEAT repeats in mammalian eIF4G [34], is largely conserved in plant eIF4G [35, 36] but is absent in yeast eIF4G [37]. eIF4A, whose RNA helicase activity unwinds secondary structure in a $5^{\prime}$-UTR to facilitate 40 $S$ subunit scanning, binds the HEAT-1/MIF4G and HEAT2/MA3 domains in mammalian eIF4G [29, 38-40]. The absence of an HEAT-2 domain in yeast eIF4G dictates that eIF4A must rely on its interaction with the HEAT-1 domain alone. HEAT-3, the third HEAT domain in mammalian eIF4G, is composed of three and one half HEAT repeats that bind Mnk eIF4E kinases 1/2 [34]. This domain is absent from plant and yeast eIF4G [37, 41] (Figure 3). 


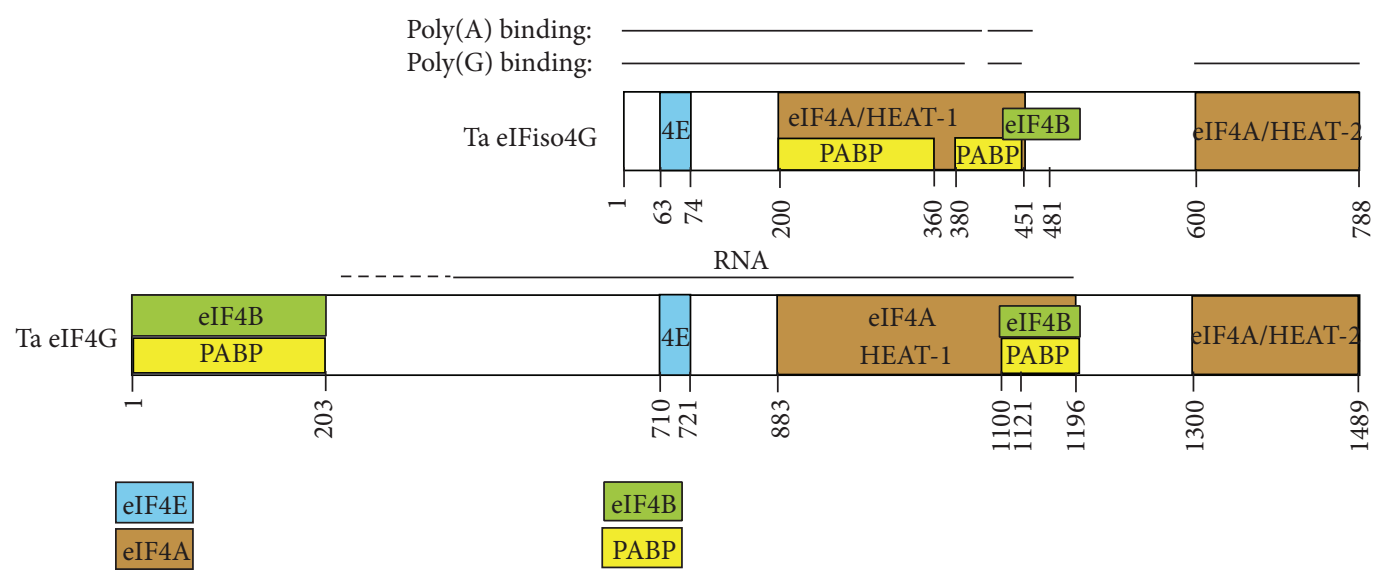

FIGURE 3: Partner protein interactions of eIF4G and eIFiso4G. The domain organizations of the RNA and protein binding domains in wheat eIF4G (TaeIF4G) and eIFiso4G (TaeIFiso4G) are shown. Each protein is drawn approximately to scale with amino acid positions indicated below each protein. Interaction domains for partner proteins are indicated in color with a key to each partner included in the figure.

We examined whether plant eIF4G was more similar to animal or yeast eIF4G by determining whether eIF4A could interact with both of its HEAT domains as in animal eIF4G or just the HEAT-1 domain as in yeast eIF4G. Using in vitro pull down assays, we demonstrated that eIF4A could bind the HEAT-2 domain as well as the HEAT-1 domain [36]. Therefore, we demonstrated that plant eIF4G is more similar to animal eIF4G than it is to yeast eIF4G in that it contains the HEAT-1/MIF4G and HEAT-2/MA3 domains, both of which bind eIF4A. Plant eIF4G is like yeast eIF4G, however, in that it lacks the HEAT-3 domain.

Proteins also interact with regions of eIF4G that lie outside its HEAT domains. eIF3 binds to a 90 amino acid region just C-proximal to the HEAT-1 domain through its c, d, and e subunits [42, 43]. eIF4A and eIF3 have been suggested to bind eIF4G in a mutually cooperative manner [44] but this was not observed in a more recent study [43]. eIF4E binds to a discrete sequence that is N-proximal to the HEAT-1 domain (Figure 3) which is conserved among animal, plant, and yeast eIF4G proteins in sequence and position (Table 1). eIF1 and eIF5 bind eIF4G, at least in yeast, within an arginine and serine rich domain that lies just Nproximal to the HEAT-1 domain and their interaction with eIF4G is mutually competitive $[45,46]$. eIF1 interacts with eIF3 and is involved in AUG selection, whereas eIF5 interacts with eIF2 and eIF3 and promotes their binding to a $40 \mathrm{~S}$ subunit as well as their function [47].

\section{5. eIFiso4G, a Highly Divergent Isoform of eIF4G, Is Unique to Plants}

Many eukaryotes express two highly similar eIF4F isoforms. In animals and yeast, the two eIF4F isoforms do not differ in the eIF4E and eIF4A subunits but they do differ in the eIF4G isoforms present which are expressed from distinct genes [37, 48]. Plants also contain two eIF4F complexes called eIF4F and eIFiso4F [49]. Whereas eIF4F is composed of eIF4E, eIF4A, and eIF4G, eIFiso4F is composed of eIFiso4E, eIF4A, and eIFiso4G [49]. eIFiso4G is smaller $(86 \mathrm{kDa})$ than plant eIF4G or eIF4G in animals or yeast and shares only limited similarity to other eIF4G proteins which is confined principally to the HEAT-1 domain (Figure 3). For example, wheat eIFiso $4 \mathrm{G}$ is only $30 \%$ identical to wheat eIF $4 \mathrm{G}$ at the protein level [41], whereas the two yeast isoforms of eIF $4 \mathrm{G}$ and the two mammalian isoforms share $53 \%$ and $46 \%$ identity, respectively $[37,48]$.

eIFiso4G contains the HEAT- 1 and HEAT-2 domains and, as with eIF4G, we demonstrated that each HEAT domain can bind eIF4A [35]. Therefore, both plant eIF4G isoforms are more similar to animal eIF4G than yeast eIF4G. We did observe, however, that eIFiso4G differs from eIF4G in that its HEAT-1 domain is sufficient to bind eIF4A [35], whereas a short region immediately C-proximal to the HEAT-1 domain of eIF4G is also required, perhaps for stable folding of the domain [36] (Figure 3).

In our analysis of the binding of eIF4A to eIFiso $4 \mathrm{G}$, we observed that deletion of the N-terminal 30-40 residues of the HEAT-1 domain abolished eIF4A interaction, while the first 40 residues of the HEAT- 1 domain were sufficient to bind eIF4A, although the binding was weak [35]. Deletions from the C-terminal end of the HEAT-1 domain progressively reduced the strength of eIF4A binding, indicating the entire domain was necessary for full binding. A seven-aminoacid sequence, that is, ILNKLTP, within the N-terminal 30 residues of the eIFiso4G HEAT-1 domain is conserved among eIF4G proteins. Its mutation in mammalian eIF4G abolished eIF4A binding [39], whereas this sequence, with the remaining portion of the HEAT-1 domain, was required for eIF4A binding [50]. The crystal structure of the yeast eIF4A:eIF4G complex demonstrated that the asparagine, lysine, and threonine residues within this sequence make direct contact with eIF4A [51]. Truncation to remove the final helix of the eIF4G HEAT-1 domain in yeast did not affect eIF4A binding but reduced its ability to enhance eIF4A ATPase activity [51]. Thus, the $\mathrm{N}$-terminal region of the HEAT-1 domain is required for the stable association of eIF4A with eIF4G. Almost all amino acid residues throughout the yeast HEAT-1 domain that contact eIF4A [51] are 
TABLE 1: The eIF4E interaction domain is conserved in eukaryotic eIF4G and 4E-binding proteins ${ }^{\mathrm{a}}$.

\begin{tabular}{lcc}
\hline Species & Protein & eIF4E binding site sequence \\
\hline Wheat & eIF4G & RKKYSRDFLLTFAH \\
Wheat & eIFiso4G & RVRYSRDQLLDLRK \\
Arabidopsis thaliana & eIF4G & EKKYSRDFLLKFAD \\
Arabidopsis thaliana & eIFiso4G1 & RVKYTREQLLELKE \\
Arabidopsis thaliana & eIFiso4G2 & RVRFSREEILQHRE \\
Homo sapiens & eIF4GI & KKRYDREFLLGFQF \\
Homo sapiens & eIF4GII & KKQYDREFLLGFQF \\
Consensus: & & $\ldots$. . RE.LL.... \\
& & D I \\
Saccharomyces cerevisiae & eIF4G1 & KYTYGPTFLLQFKD \\
Saccharomyces cerevisiae & eIF4G2 & KYTYGPTFLLQFKD \\
Saccharomyces cerevisiae & P20 & MIKYTIDELFQLKP \\
Homo sapiens & $4 \mathrm{E}-\mathrm{BP} 1$ & RIIYDRKFLMECRN \\
Homo sapiens & $4 \mathrm{E}-\mathrm{BP} 2$ & RIIYDRKFLLDRRN \\
Homo sapiens & $4 \mathrm{E}-\mathrm{BP} 3$ & RIIYDRKFLLECKN
\end{tabular}

${ }^{\mathrm{a}}$ Sequence of the eIF4E binding site in wheat, Arabidopsis thaliana, human, and yeast eIF4G is shown as is sequence of the eIF4E binding site in the $4 \mathrm{E}$ binding proteins from yeast (Sc p20) and human (Hs4E-BP). The consensus is derived from eIF4G proteins in higher eukaryotes.

conserved in eIFiso4G. Plant eIFiso4G and eIF4G differ from yeast eIF4G, however, in that they lack a tryptophan residue that contributes to yeast eIF4A binding [51], consistent with our observation that this region is not required for eIF4A binding to eIFiso4G.

Residues in the mammalian HEAT-2 domain that contact eIF4A are present in the wheat eIFiso4G HEAT-2 domain [52]. Plant eIFiso4G and eIF4G, however, lack the sequence corresponding to $\alpha$-helices H7b, H8, and $\mathrm{H} 9$ of the mammalian HEAT-2 domain, indicating significant divergence from the mammalian homolog. As eIF4A is typically lost from eIF4G and eIFiso4G during their purification [49], the strength of eIF4A binding may be lower than that in animal eIF4G. Nevertheless, we observed that eIF4A binds the HEAT-1 and HEAT-2 domains of eIFiso4G and eIF4G $[35,36]$.

\section{PABP Interacts with eIF4G and eIFiso4G in Distinct Ways}

As eIF4G and eIFiso4G are similar in that both contain two HEAT domains but differ dramatically in mass, I was particularly interested in discerning the basis for the interaction of PABP with each. To examine this, I attempted to use the yeast two-hybrid interaction assay but like laboratories working with the animal or yeast homologs, I found the interaction too weak to detect with this approach. Therefore, we used an in vitro pull-down assay in which the interaction could be reliably detected. Although only a single interaction domain for PABP has been reported in animal and yeast eIF4G [2731], we discovered that PABP binds to two distinct sites within plant eIF4G [36]. The first of these lies N-proximal to the eIF4E interaction domain in eIF4G (Figure 3) and this interaction site is conserved in position with animal and yeast eIF4G [27-31, 36, 53]. We determined that the second PABP binding site lies C-proximal to the HEAT-1 domain which appears to be unique to plant eIF4G and represents a significant difference among eIF4G homologs (Figure 3) [36]. Whether PABP can bind to the corresponding region in animal and yeast eIF4G, however, warrants reexamination.

The smaller size of eIFiso4G suggested to me that there might be a substantial difference in its domain organization. Even before I began the analysis, I knew that because eIFiso4G lacks most of the region N-terminal to the HEAT1 domain present in eIF4G, not only does the eIFiso4E interaction site lie closer to the eIFiso4G N-terminus than does the eIF4E interaction site in eIF4G, but also the region corresponding to the $\mathrm{N}$-terminal PABP interaction domain of eIF4G is entirely missing in eIFiso4G. Our analysis of PABP binding to eIFiso4G revealed that PABP binds within the HEAT-1 domain at two contiguous sites which overlap extensively with the eIF4A binding site (Figure 3) [35]. The proximity of the two PABP binding sites may actually represent a single interaction domain containing more than one subregion capable of supporting PABP binding. Because the eIFiso4E interaction site lies so close to the N-terminus of eIFiso4G, PABP binds to the C-proximal side of the eIF4E binding site (Figure 3), a significant difference from animal and yeast eIF4G where PABP binds N-proximal to the eIF4E binding site. Therefore, we were able to show that eIFiso4G is unlike plant eIF4G in that it binds PABP at a single site and is unlike any other known eIF4G protein in that this single site encompasses the HEAT-1 domain [35].

\section{7. eIF4B: An Alternative Bridge Linking PABP and the Cap-Binding Complex in Higher Eukaryotes}

eIF4B stimulates the RNA-dependent ATP hydrolysis activity and the ATP-dependent RNA helicase activity of animal eIF4A [7-15], the helicase activity of yeast eIF4A [54], and the 
activity of plant eIF4A [55-58]. eIF4B increases the affinity of eIF4A for ATP and its helicase processivity [59, 60]. As such, our discovery of an interaction between PABP and eIF4B was surprising as no direct interaction between eIF4B and eIF4G or eIF4A had been reported for any eukaryote. Moreover, because so little was known about the interaction of eIF4B with any component of the cap-binding complex, it was not an obvious candidate for mediating the interaction between the termini of an mRNA. However, eIF4B mediates mRNA binding to ribosomes [61-65], suggesting that PABP may interact with this factor to promote ribosomal binding to an mRNA. This prompted me to investigate the molecular basis for the interaction between PABP and eIF4B.

As with the interaction between PABP and eIF4G, the interaction between PABP and eIF4B is too weak to detect using the yeast two-hybrid assay. However, in vitro pulldown analysis revealed that PABP binds to two sites in eIF4B that are conserved repeats approximately 22 amino acids long and that the two repeats lie on opposite sides of a Cterminal RNA binding domain [66] (Figure 4). Interestingly we observed that eIF4A binds N-proximal to each PABPbinding repeat to a conserved 18-amino-acid long repeat, suggesting that eIF4B may bind two molecules each of PABP and eIF4A. These two repeats are conserved among plant eIF4B proteins and to a limited extent in human and yeast eIF4B $[66,67]$, suggesting this region in the human and yeast homologs may play a similar role. Following our discovery of an interaction between PABP and eIF4B, the PABP binding site in mammalian eIF4B was reported to lie within the Nterminal 80 amino acids [32]. Despite the direct interaction between eIF4B and PABP demonstrated for the plant and animal homologs, no such interaction has been reported in yeast. Our findings, corroborated in animals, enabled us to conclude that eIF4B contributes to the assembly of the translation initiation machinery onto an mRNA and likely plays a role in mediating the interaction between the termini of an mRNA.

Our discovery that eIF4A binds proximal to PABP at two sites within eIF4B suggested to me a possible functional interaction between the partner proteins, a possibility supported by the observation that PABP increases the ATPase and the RNA helicase activities of the plant cap-binding complex [17]. The observation that PABP and eIF4A bind on either side of the eIF4B C-terminal RNA binding domain also suggests that they may affect the activity of this RNA binding domain. Thus, the proximity of PABP to eIF4A when bound to eIF4B may be needed for PABP to facilitate eIF4A activity.

Although eIF4B can bind PABP, eIF4B is not known to bind the $5^{\top}$-cap, and therefore its ability to act as a bridge between the termini of an mRNA requires that it interacts with one or more components of the cap-binding complex. As eIF4A is more abundant than eIF4G, I realized it was important to examine whether eIF4B could interact with another subunit of the cap-binding complex in addition to eIF4A if it were going to mediate an interaction between the cap-binding complex and PABP. Although no direct interaction between eIF4B and eIF4G had been reported in animals or yeast, we discovered that eIF4B does bind eIF4G and eIFiso $4 \mathrm{G}$ directly $[35,36]$. We then undertook to identify the eIF4B binding site in eIF4G and discovered that it bound to nearly the same regions responsible for binding PABP: the first within the N-terminal region of eIF4G and the second lying just C-proximal to the HEAT-1 domain (Figure 3) [36].

eIF4B also binds eIFiso4G, but, as with PABP, the absence of a region corresponding to the $\mathrm{N}$-terminal region of eIF4G precluded the possibility that eIF4B could bind eIFiso $4 \mathrm{G}$ at sites similar to those in eIF4G. Instead, our analysis of their interaction revealed that eIF4B bound eIFiso $4 \mathrm{G}$ at a single site that overlaps the C-terminal end of the HEAT-1 domain and overlaps the C-terminal end of the PABP binding site within the HEAT-1 domain [35] (Figure 3).

When we mapped where eIFiso4G bound eIF4B, we found that it binds at a single site proximal to the $\mathrm{N}$-terminal RNA binding domain of eIF4B [66] (Figure 4). The binding site of eIF4G in eIF4B has not been delineated in detail although it lies within the N-terminal region of eIF4B [36] but whether eIF4G binds precisely at the same site that eIFiso4G does remains to be determined. Thus, the direct binding of eIF4B to either eIF4G or eIFiso $4 \mathrm{G}$ provides the link with the cap-binding complex that eIF4B would require for it to act as a bridge between the cap-binding complex and PABP. Additionally, as eIF4A is typically lost during the purification of eIF4G or eIFiso4G [49], the ability of eIF4B to bind eIF4G or eIFiso4G and to interact with eIF4A $[35,66]$ may stabilize eIF4A within the eIF4F and eIFiso4F complexes.

\section{8. eIF4B and PABP Compete for Binding to eIF4G and eIFiso4G}

Our discovery that eIF4B and PABP bind to two independent and similar regions of eIF4G raised the intriguing possibility that they bind either cooperatively or competitively to each region. We therefore employed eIF4G binding assays in which one partner protein is held at a constant concentration while the second partner protein is added in increasing amounts to examine its effect on the binding of the first partner. The results from these assays showed that eIF4B and PABP bind competitively to each region of eIF4G [36], suggesting that the binding of PABP and eIF4B to these sites is mutually exclusive. When PABP and either region of eIF4G were present in equal molar amounts in the binding assay, eIF4B competed effectively with PABP, even at substoichiometric molar ratios, suggesting that eIF4B binds eIF4G at each site more strongly than does PABP. Because eIF4B more effectively competed against PABP at the HEAT1-proximal binding site than at the N-proximal binding site [36], eIF4B may bind relatively more strongly to the HEAT1-proximal site than the N-proximal site.

Because the binding sites for PABP and eIF4B overlap extensively in eIFiso4G, we also tested whether they compete for binding eIFiso4G using the same approach and observed that in fact they do compete for binding eIFiso4G just as they do for eIF4G $[35,36]$. This competition suggests the binding of PABP and eIF4B to eIFiso4G or eIF4G is mutually exclusive and they would not be expected to bind the same molecule of eIFiso4G or eIF4G simultaneously. 

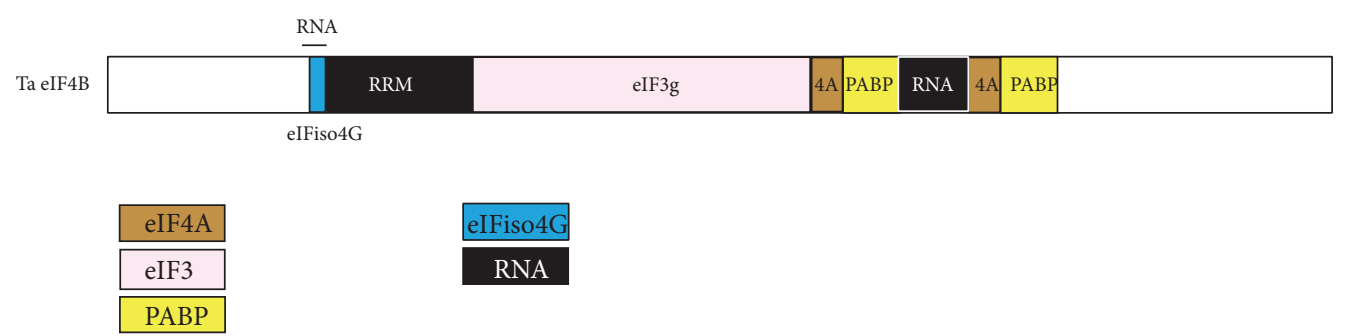

FIGURE 4: Partner protein interactions of eIF4B. Domain organization for RNA and partner protein binding sites in wheat eIF4B (TaeIF4B). The protein is drawn approximately to scale and interaction domains for partner proteins are indicated in color with a key to each partner included in the figure.

\section{The Two HEAT Domains in eIF4G and eIFiso4G Enable Simultaneous Binding of eIF4B and EIF4A or EIF4A and PABP}

Because the binding sites for eIF4B and PABP partially overlap with the eIF4A binding domain in the HEAT-1 domain of eIF4G, we investigated whether they would compete with eIF4A in binding eIF4G. Our competition assays revealed, however, that eIF4B or PABP did not alter binding of eIF4A to the eIF4G HEAT-1 domain whether or not the HEAT-2 domain was also present [36], suggesting that the eIF4B or PABP binding sites do not overlap sufficiently with the eIF4A interaction domain to compete with eIF4A in binding eIF4G. Similarly, eIF4A had no detectable effect on the binding of eIF4B or PABP to the eIF4G HEAT-1 domain. I concluded, therefore, that eIF4A and either eIF4B or PABP can bind eIF4G simultaneously but that eIF4B and PABP compete for binding to each site in eIF4G.

Given the results with eIF4G, it was surprising when we observed that PABP competes with eIF4A for binding to the eIFiso4G HEAT-1 domain [35]. eIF4B also competes with eIF4A for binding to the eIFiso4G HEAT-1 domain [35]. The PABP binding site overlaps the eIF4A binding site extensively in the eIFiso4G HEAT-1 domain while the eIF4B binding site partially overlaps the eIF4A binding site. However, when a portion of eIFiso4G containing the HEAT-1 and HEAT-2 domains was used in the competition assay, no competition between PABP and eIF4A or between eIF4B and eIF4A was observed [35]. Thus, in the absence of the HEAT-2 domain, binding of eIF4A to the eIFiso4G HEAT-1 domain may be weaker and more likely to be displaced by PABP or eIF4B. The eIFiso4G HEAT-2 domain, which also binds eIF4A, likely alleviates the competition perhaps through stabilizing eIF4A binding to eIFiso4G in the presence of PABP. Despite our observations with the eIFiso4G HEAT-1 domain alone, the binding of PABP or eIF4B to full-length eIFiso4G would not be expected to compete with eIF4A due to the presence of the HEAT-2 domain. Therefore, no difference in the interaction of these partner proteins with eIFiso $4 \mathrm{G}$ or eIF $4 \mathrm{G}$ would be predicted.

\section{0. eIF4G, eIFiso4G, and eIF4B Bind Competitively to PABP}

PABP contains four, N-terminal RNA recognition motifs (RRMs) that are tandemly arranged and a C-terminal region

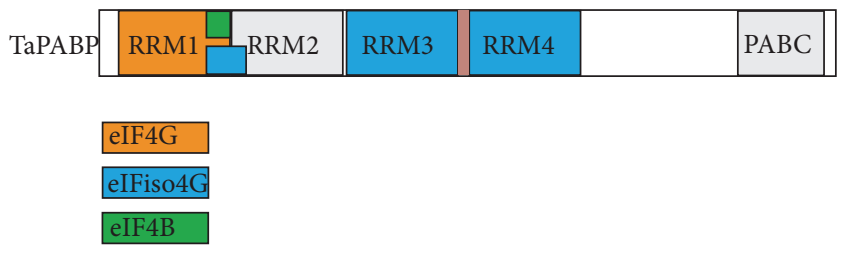

FIGURE 5: Partner protein interactions of PABP. Domain organization for RNA and partner protein binding sites in wheat PABP (TaPABP). The protein is drawn approximately to scale and interaction domains for partner proteins are indicated in color with a key to each partner included in the figure.

involved in self-association $[68,69]$. The region encompassing RRM1 to RRM2 in PABP is required for interaction with mammalian eIF4G whereas PABP RRM2 is principally required for binding yeast eIF4G although PABP RRM1 also contributes to the binding $[29,70,71]$. In our analysis of the plant homologs, we discovered that eIF4G binds within RRM1 of PABP and eIFiso4G binds a region encompassing the C-terminal end of RRM1 and the N-terminal end of RRM2 [72] (Figure 5), suggesting that eIFiso4G binds to a region in $\mathrm{PABP}$ that roughly corresponds to the region in mammalian PABP required for eIF4G binding.

In addition to its principal binding site spanning the $\mathrm{C}$ terminal end of RRM1 and the N-terminal end of RRM2, eIFiso4G interacts weakly with RRM3-4 [72] (Figure 5). As mentioned above, PABP binds to two distinct subsequences within the eIFiso4G HEAT-1 domain [35] (Figure 3). PABP RRM3-4 binds to the N-terminal portion of the HEAT-1 domain, whereas the region of PABP encompassing the Cterminal end of RRM1 and the N-terminal end of RRM2 binds the C-terminal portion of the HEAT-1 domain [35]. The only PABP binding site identified to date in animal and yeast eIF4G, however, lies upstream of the eIF4E binding site [2830 ] and eIF4B has been reported to bind mammalian PABP within its C-terminal region that also interacts with eRF3, Paip1, and Paip2 [32, 73-77]. All known proteins that bind the PABP C-terminal region harbor a PAM2 motif but no PAM2 motif is present in mammalian eIF4B. Moreover, the PABP C-terminal region of plant PABP interacts with PAM2 motifcontaining proteins such as ERD15, PCI6, PCI243, and CID7 but not eIF4B [72, 78-80].

Our analysis of the binding of eIF4B to PABP revealed that it interacted with the RRM1 of PABP at a site that 
substantially overlaps with the eIFiso4G binding site. Not surprisingly, we found that eIF4B and eIFiso4G compete in their binding to PABP, resulting in mutually exclusive binding to PABP [72] (Figure 5), data that are consistent with the mutually exclusive binding of eIF4B and PABP to eIFiso4G [35]. I interpreted these results to mean that a molecule of PABP can bind either eIF4B or eIFiso4G but not both simultaneously just as a molecule of eIFiso4G can bind either eIF4B or PABP but not both simultaneously. Physiologically relevant concentrations of zinc promote the homodimerization of eIF4B and increase the strength of the eIF4B-PABP interaction such that eIFiso4G is unable to compete with eIF4B in binding PABP which it can do in the absence of zinc [81]. However, zinc does stabilize the interaction between eIF4B and eIFiso4G, albeit to a lower extent [81].

Interestingly, our analysis of the binding domains of eIF4B showed that eIFiso4G binds to a short region near its $\mathrm{N}$-terminus, whereas PABP binds to two repeats in the central region of eIF4B as mentioned above [66] (Figure 4). These findings suggest that eIFiso $4 \mathrm{G}$ and PABP may be able to bind eIF4B simultaneously, thus enabling it to serve as a bridge between the cap-binding complex and PABP.

\section{Functional Consequences of the Interactions between PABP and the Cap-Binding Complex}

The fact that PABP and the cap-binding complex interact raises the question of how their physical interaction affects their function. My observation that the $5^{\prime}$-cap and the poly(A) tail stimulate translation synergistically, correlating with the physical interactions between PABP bound to the poly (A) tail and the cap-binding complex bound at the $5^{\prime}$ cap $[19,82]$, suggested that an increased yield of protein from a given mRNA is the primary outcome of the interactions between the termini of the mRNA. But I wanted to know how exactly this increase in translatability was achieved.

One functional consequence of the physical interaction between PABP and eIFiso4G is that PABP increases the binding of eIF4F or eIFiso4F to the $5^{\prime}$-cap [16], similar to observations made with the homologs in yeast [83]. PABP also stimulates the ATPase and RNA helicase activity of eIFiso4F when eIF4A and eIF4B are present [17]. In mammals, the results have been mixed. Depletion of PABP from cell extracts impaired the binding of eIF4E to the $5^{\prime}$ cap [84] but deletion of the N-terminal PABP-binding site in eIF4G had no effect on the interaction between eIF4G and eIF4E, at least when tested in vitro [85].

Addition of eIF4B to eIFiso4F or to the eIFiso4F-PABP complex in plants lowered the activation energy of binding of each to the $5^{\prime}$-cap $[86,87]$ which fluorescence stopped-flow studies suggested resulted from a concentration-independent conformational change and a reduced dissociation rate [86]. Thus, PABP and eIF4B enhance eIFiso4F binding to a $5^{\prime}$ cap by means of a conformational change propagated to the cap binding site that enables eIFiso4F to achieve more stable binding to the $5^{\prime}$-cap. This can also explain how PABP increases the ATPase and RNA helicase activity of the capbinding complex [17]. The interaction between PABP and eIF4G stimulates eIF4E cap binding in animals [84] and yeast [88], perhaps by assisting eIF4G to outcompete general RNAbinding proteins that are unable to interact with PABP [89].

While PABP increases the binding of the cap-binding complex to a $5^{\prime}$-cap, we showed that eIFiso4G, eIF4G, or eIF4B increases PABP binding to poly(A) RNA through a decrease in the dissociation rate of $\mathrm{PABP}[27,53]$. The synergistic effect that eIF4F (or eIFiso4F) and eIF4B have on the binding activity of PABP to poly(A) RNA [27] supports a functional interaction among eIF4G, eIF4B, and PABP (or eIFiso4G, eIF4B, and PABP). The actual circularization of an mRNA predicted by the physical interaction between eIF4G and PABP when bound to their respective mRNA sites was shown for the yeast homologs [90].

The interaction between PABP and eIF4G promotes formation of the $48 \mathrm{~S}$ preinitiation complex and the assembly of the $80 \mathrm{~S}$ ribosome $[84,85,91,92]$, which correlates with the stimulatory role that the $\mathrm{PABP} /$ poly(A) tail plays during translation [19]. Thus, one way PABP promotes translation is to increase $40 \mathrm{~S}$ subunit binding to an mRNA through increasing the binding of the cap-binding complex. An additional consequence of the physical interactions between PABP and the cap-binding complex is that these interactions serve as a means test to confirm the integrity of an mRNA prior to $40 \mathrm{~S}$ subunit recruitment [93]. In other words, if the PABP bound to the poly $(\mathrm{A})$ tail is involved in promoting translation through its interaction with the cap-binding complex bound at the $5^{\prime}$-cap, both ends of an mRNA are confirmed to be present, ensuring that translation does not initiate on an mRNA that is actively undergoing degradation.

Yet another consequence of the interaction between PABP and the cap-binding complex results from the competition between eIF4G (or eIFiso4G) and eIF4B in binding PABP. With a packing density of one PABP per 27 residues in animals and 25 residues in yeast $[68,94]$ and an average poly(A) tail length of approximately 150 or 60 adenosine residues for newly synthesized and processed mRNAs in animals or yeast, respectively, multiple molecules of PABP would be expected to bind a poly $(\mathrm{A})$ tail. The presence of multiple molecules of PABP on an mRNA provides multiple targets for interaction for the cap-binding complex. As eIF4G (or eIFiso4G) and eIF4B can interact with PABP, their mutually exclusive binding to $\mathrm{PABP}$ increases the number of possible interactions with separate molecules of PABP on a poly(A) tail, providing increased stability to the $\mathrm{PABP} / \mathrm{cap}$ binding complex through an increase in the number of protein interactions.

Because eIF4B can dimerize and each eIF4B has two binding sites for PABP, eIF4B may be able to increase the number of interactions with PABP bound to the poly(A) tail and thus stabilize the physical interactions between the termini of an mRNA even further. Given the multiple possible interactions among eIF4G (or eIFiso4G), eIF4B, and PABP, more than one type of interaction may occur during translation initiation. Individual molecules of PABP along a poly(A) tail may interact directly with eIF4G/eIFiso4G and eIF4B. Alternatively, a molecule of PABP may interact with 
eIF4B only while the same eIF4B (either in its monomer or dimer form) interacts with eIF4G (or eIFiso4G), thus acting as a bridge between these proteins. The interaction between eIF4B and eIF3 $[95,96]$ provides yet another means to link PABP with eIF4G as mediated by eIF4B and eIF3. We also demonstrated that PABP forms homomeric complexes upon binding to poly(A) RNA $[27,53]$ in agreement with PABP in animals [69]. As mentioned, the binding of eIF4G (or eIFiso4G) and eIF4B to PABP promotes its homomeric binding to poly(A) RNA $[27,53]$ and this would be expected to increase further the stability of the PABP/cap-binding complex. The observation that the combination of eIF4G (or eIFiso4G) and eIF4B synergistically promotes PABP binding to poly(A) RNA and does so in part through promoting multimeric PABP binding [27] suggests that eIF4G (or eIFiso4G) and eIF4B cooperate to interact with PABP and increase the stability of the complex.

\section{Regulation of the Interaction between the Cap-Binding Complex and PABP}

Our analysis of PABP and the cap-binding complex revealed that both of them are subject to phosphorylation, and, in the case of PABP, its phosphorylation state determines its type of binding and affinity to poly(A) RNA [53]. PABP is differentially phosphorylated in plants as it is in yeast and sea urchin $[53,97-99]$. We found that phosphorylated isoforms of PABP bind $\operatorname{poly}(\mathrm{A})$ RNA cooperatively, whereas hypophosphorylated PABP isoforms bind poly(A) RNA noncooperatively but with greater affinity [53]. However, cooperative binding is greatest between phosphorylated and hypophosphorylated isoforms of the protein $[53,100]$, suggesting that the multiple molecules of PABP bound along the average poly (A) tail likely present a heterogeneous population of phosphorylated states, consistent with what is observed for polysome-bound PABP isolated from actively growing plant tissues [53].

We also discovered that the phosphorylation state of PABP determines the strength and specificity of its interaction with components of the cap-binding complex [53]. eIF4G interacts with hypophosphorylated PABP specifically and increases its RNA binding by promoting its cooperative binding to poly(A) RNA while eIFiso4G interacts with PABP independent of its phosphorylation state [53]. eIF4B increases the RNA binding activity of phosphorylated PABP to a greater extent than hypophosphorylated PABP through an increase in RNA affinity [53], suggesting that an interaction between phosphorylated PABP and eIF4B is preferred.

When we examined the phosphorylation state of eIF4B, we found that it determines the strength and specificity of its interaction with PABP. Phosphorylated eIF4B isoforms increased the RNA binding activity of phosphorylated PABP, whereas recombinant eIF4B, lacking phosphorylation, had little effect [53]. As eIF4B has a greater effect on the RNA binding activity of PABP than does eIF4F or eIFiso4F [27], eIF4B may contribute substantially to stabilizing the interaction between the cap-binding complex and PABP.

The overlapping nature of the eIF4G, eIFiso4G, and eIF4B binding sites in PABP forces eIF4G and eIF4B (or eIFiso4G and eIF4B) to interact with different molecules of PABP. The fact that the phosphorylation state of PABP determines whether it will interact with eIF4G or eIF4B serves to prevent eIF4G and eIF4B (or eIFiso4G and eIF4B) from competing in binding the same PABP molecule. This suggested to us that two molecules of PABP are needed to interact with eIF4G and eIF4B (or eIFiso4G and eIF4B) simultaneously. As this would involve interactions among four molecules (i.e., eIF4G, eIF4B, and two molecules of PABP) instead of three (i.e., eIF4B, eIF4G, and a single molecule of PABP), it would provide greater stability to the complex. This conclusion is consistent with our observation that eIF4G and eIF4B (or eIFiso4G and eIF4B) synergistically promote multimeric binding of PABP to poly(A) RNA $[27,53]$. As eIF4B binds eIF4G (or eIFiso4G) and eIF3 while PABP isoforms can interact when bound to poly(A) RNA [35, 36, 53, 96], these additional interactions would increase the stability of the PABP-cap-binding complex even further.

Following our observation that the phosphorylation state of eIF4B and PABP determines the specificity and strength of their interactions, I asked whether their interaction might be subject to regulation through changes in their phosphorylation state. We have not observed that the phosphorylation state of PABP changes significantly during development or following exposure to stress $[98,100]$. The phosphorylation state of eIF4B, however, is highly dynamic and changes in response to developmental or stress-related cues. eIF4B is present as phosphorylated isoforms during active translation in growing tissues and it is these isoforms that are preferentially recruited into polysomes $[53,98]$. In contrast, eIF4B is present in its dephosphorylated isoforms during late seed development or following a heat shock, conditions under which protein synthesis is repressed [98]. Regulation of eIF4B phosphorylation and therefore its interaction with PABP as well as any other roles that phosphorylation of eIF4B may play could be one means by which global protein synthesis is controlled. Interestingly, the phosphorylation state of mammalian eIF4B is similarly regulated during growth or following stress [101-103] but whether the interaction between mammalian eIF4B and PABP is regulated by the phosphorylation state of eIF4B has not been examined.

\section{Remaining Questions and Future Directions}

With these results, we can see how our view of mRNAs has been transformed from a linear and rigid model to one that is circular with the termini held in close physical proximity through interactions mediated by PABP and the cap-binding complex. Despite the longstanding knowledge that mRNA is single-stranded, flexible, and capable of intramolecular base pairing, it is probable that depictions of mRNA in the scientific literature as straight molecules had influenced thinking against considering that the $3^{\prime}$-end might be involved in translation initiation. Only with empirical evidence demonstrating a synergistic functional interaction between a cap and a poly (A) tail [19], did the notion of cooperation between $\mathrm{PABP}$ and the cap-binding complex begin to transform our 
thinking about the role of PABP in protein synthesis. As with any good discovery, more questions are raised than answered. The first and foremost of these is how important is the interaction to protein synthesis?

Reports addressing this question have varied widely in their answers $[65,84,85,104,105]$. Some have concluded that disrupting the PABP-eIF4G interaction is not essential for translation but may contribute to optimal translation. For example, depletion of PABP from extracts reduced eIF4E binding to a cap, $48 \mathrm{~S}$ complex formation, and translation, all of which could be restored by the addition of wild-type PABP but not by a mutant of PABP unable to interact with eIF4G [84]. However, deletion of the PABP binding site from eIF4GI had little effect on the latter's ability to support translation in vivo although the siRNA knockdown approach used to suppress endogenous eIF4G expression in vivo may not have been stringent enough to assay fully the contribution that the PABP-eIF4G interaction has on translation [85]. Deletion of the PABP binding site in eIF4G did reduce its efficiency of incorporation into polysomes following a hypertonic shock [85]. Whether the interaction between PABP and eIF4B, which may have stabilized the association of PABP with the cap-binding complex even when the interaction between PABP and eIF4G was disrupted, masked the true contribution that the PABP-eIF4G interaction makes to translation was not considered in these studies. The many interactions among PABP, eIF4G, eIF4B, eIF4A, eIF4E, and eIF3 make likely the possibility of functional redundancy in the complex and in the interaction with PABP. In this view, no interaction is solely responsible but each contributes to the overall rate of assembly and stability of the complex. Without disrupting all avenues for interaction between PABP and the cap-binding complex, however, it is impossible to estimate the magnitude that this interaction makes to translation and whether it might differ in animals, plants, and yeast.

The conditions under which the contribution that the interaction between PABP and the cap-binding complex makes to translation also can influence measurements of the importance, or lack thereof, of the interaction. For example, the PABP-eIF4G interaction appears to be more important under conditions of competitive translation, that is, when the amount of cap-binding complex or PABP is not in excess or in the presence of general ribonucleoproteins. For example, the cap dependency of translation in wheat germ lysate is inversely proportional to the concentration of PABP or eIF4G/eIFiso4G [41]. Moreover, the presence of a general ribonucleoprotein such as YB-1 allows only proteins with sufficient strength and specificity to compete for mRNA binding which the combined strength of eIF4G and PABP can do [89]. Thus, the combinatorial strength provided by the multiple interactions among PABP, eIF4G, eIF4B, eIF4A, eIF4E, and eIF3 is mostly likely important in the environment of the cell in which the full complement of RNA binding proteins and mRNAs is present.

Another question regards the contribution that the interaction between PABP and eIF4B makes to the assembly of the cap-binding complex, $48 \mathrm{~S}$ complex formation, and translation. We have shown that eIF4B interacts with PABP and promotes its multimeric binding to poly(A) RNA [27,
$53,100]$. In animals, however, other than the report that PABP and eIF4B can interact [32], little has been done to investigate what function this interaction has. Nevertheless, the observation that the interaction is conserved in animals and plants does suggest that it serves a function, at least in higher eukaryotes. That the interaction between PABP and eIF4B is conserved in plants and animals is even more compelling given that eIF4B is the least conserved of all the initiation factors. Future work will need to examine whether eIF4B promotes the multimeric binding of PABP to poly(A) RNA in animals and whether the PABP-eIF4B interaction promotes $48 \mathrm{~S}$ complex formation and translational activity.

Related questions concerning the role of eIF4B in the interaction also require addressing. For example, as eIF4B contains two binding sites for PABP and eIF4A, does eIF4B bind one or two molecules of PABP and eIF4A and what functional consequences result from the proximity of their binding to each site in eIF4B? Similarly, although the binding of PABP and eIF4B to eIFiso4G is mutually exclusive, plant eIF4G contains two PABP and eIF4B binding sites, to which PABP and eIF4B bind competitively. Future work will need to address whether PABP and eIF4B can bind the same molecule of eIF4G simultaneously and if not whether PABP and eIF4B cycle through eIF4G during translation initiation.

Perhaps the most intriguing question of all that remains unanswered is why does translation benefit from an interaction between the termini of an mRNA? Even if PABP increases the assembly of the cap-binding complex at a $5^{\prime}$ cap in order to promote ribosome recruitment and translation initiation, surely the cap-binding complex could have evolved to bind a $5^{\prime}$-cap without assistance from PABP. The observation that many mRNAs naturally lacking a $5^{\prime}$-cap or a poly(A) tail still retain a requirement for an interaction between the termini of an mRNA [21-26, 30] indicates that this is not a specific requirement of the cap-binding complex and PABP but rather a common feature among mRNAs. The observation that the synergistic contribution that a poly (A) tail makes to the translation of a capped mRNA is not first detected until after the first round of translation [106] suggests that PABP may promote the rerecruitment of terminating ribosomes. Whether the interaction between the cap-binding complex and PABP is involved in the release of a ribosome from an mRNA prior to its rerecruitment is a question worth examining.

The circularization of an mRNA resulting from an interaction between the cap-binding complex and PABP raises the question of whether a bona fide circular mRNA would be as efficiently translated as it would seem to obviate the need for these interacting proteins to facilitate mRNA circularization. However, from the perspective of a terminating ribosome, a circular mRNA is functionally similar to a dicistronic (or polycistronic) mRNA in that the initiation codon of the single open reading frame in a circular mRNA lies downstream of a terminating ribosome. Except in cases where the $5^{\prime}$ cistron is extremely short or when certain viral proteins that promote reinitiation are present, reinitiation at distal cistrons of dicistronic or polycistronic mRNAs is typically extremely poor. This is due to the fact that a ribosome remaining bound to an mRNA following termination must regain competency 
in order to recognize a downstream initiation codon and this requires the recruitment of a ternary complex (eIF2tRNAi-GTP) and other initiation factors (e.g., eIF1, eIF1A, and eIF3) needed for initiation codon recognition. Through its interaction with the initiation factors bound to a free 40 $\mathrm{S}$ subunit (i.e., a $43 \mathrm{~S}$ complex), the cap-binding complex recruits only $40 \mathrm{~S}$ ribosomal subunits to an mRNA that are competent. To the extent that the interaction between the cap-binding complex and PABP promotes rerecruitment of a terminating ribosome suggests that these proteins may also promote restoring its competency in order to participate in another round of initiation, although this has yet to be examined. Whether the interaction between the termini of an mRNA serves as a means test to ensure the structural integrity of an mRNA prior to its translation, whether it serves to promote first-round translation, and/or whether it serves to coordinate initiation with termination through the rerecruitment of ribosomes that have completed translation will be fascinating areas of interest for future research.

\author{
Abbreviations \\ eIF: $\quad$ Eukaryotic initiation factors \\ HEAT: Huntington, elongation factor 3, PR65/A, TOR \\ PABP: Poly(A)-binding protein \\ RRM: RNA recognition motif \\ 5'-UTR: $5^{\prime}$-untranslated region.
}

\section{Conflict of Interests}

The author declares that there is no conflict of interests regarding the publication of this paper.

\section{Acknowledgments}

This work was funded by a Grant from the National Science Foundation (DBI-0820047), the USDA, and the University of California Agricultural Experiment Station.

\section{References}

[1] J. Shine and L. Dalgarno, "The $3^{\prime}$ terminal sequence of Escherichia coli $16 \mathrm{~S}$ ribosomal RNA: complementarity to nonsense triplets and ribosome binding sites," Proceedings of the National Academy of Sciences of the United States of America, vol. 71, no. 4, pp. 1342-1346, 1974.

[2] M. Kozak, "At least six nucleotides preceding the AUG initiator codon enhance translation in mammalian cells," Journal of Molecular Biology, vol. 196, no. 4, pp. 947-950, 1987.

[3] T. Preiss and M. W. Hentze, "Starting the protein synthesis machine: eukaryotic translation initiation," BioEssays, vol. 25, no. 12, pp. 1201-1211, 2003.

[4] L. D. Kapp and J. R. Lorsch, "The molecular mechanics of eukaryotic translation," Annual Review of Biochemistry, vol. 73, pp. 657-704, 2004.

[5] T. V. Pestova, J. R. Lorsch, and C. U. T. Hellen, “The mechanism of translation initiation in eukaryotes," in Translational Control in Biology and Medicine, M. B. Mathews, N. Sonenberg, and J.
W. B. Hershey, Eds., pp. 87-128, Cold Spring Harbor Laboratory Press, 2007.

[6] D. R. Gallie, "Protein-protein interactions required during translation," Plant Molecular Biology, vol. 50, no. 6, pp. 949-970, 2002.

[7] B. K. Ray, T. G. Lawson, J. C. Kramer et al., "ATP-dependent unwinding of messenger RNA structure by eukaryotic initiation factors," The Journal of Biological Chemistry, vol. 260, no. 12, pp. 7651-7658, 1985

[8] R. D. Abramson, T. E. Dever, T. G. Lawson, B. K. Ray, R. E. Thach, and W. C. Merrick, "The ATP-dependent interaction of eukaryotic initiation factors with mRNA.," Journal of Biological Chemistry, vol. 262, no. 8, pp. 3826-3832, 1987.

[9] T. G. Lawson, K. A. Lee, M. M. Maimone et al., "Dissociation of double-stranded polynucleotide helical structures by eukaryotic initiation factors, as revealed by a novel assay," Biochemistry, vol. 28, no. 11, pp. 4729-4734, 1989.

[10] S. C. Milburn, J. W. B. Hershey, M. V. Davies, K. Kelleher, and R. J. Kaufman, "Cloning and expression of eukaryotic initiation factor 4B cDNA: sequence determination identifies a common RNA recognition motif," EMBO Journal, vol. 9, no. 9, pp. 27832790, 1990.

[11] F. Rozen, I. Edery, K. Meerovitch, T. E. Dever, W. C. Merrick, and N. Sonenberg, "Bidirectional RNA helicase activity of eucaryotic translation initiation factors $4 \mathrm{~A}$ and $4 \mathrm{~F}$," Molecular and Cellular Biology, vol. 10, no. 3, pp. 1134-1144, 1990.

[12] M. Jaramillo, T. E. Dever, W. C. Merrick, and N. Sonenberg, "RNA unwinding in translation: assembly of helicase complex intermediates comprising eukaryotic initiation factors eIF- $4 \mathrm{~F}$ and eIF-4B," Molecular and Cellular Biology, vol. 11, no. 12, pp. 5992-5997, 1991.

[13] M. Altmann, P. P. Muller, B. Wittmer, F. Ruchti, S. Lanker, and H. Trachsel, "A Saccharomyces cerevisiae homologue of mammalian translation initiation factor $4 \mathrm{~B}$ contributes to RNA helicase activity," The EMBO Journal, vol. 12, no. 10, pp. 39974003, 1993.

[14] N. Méthot, A. Pause, J. W. B. Hershey, and N. Sonenberg, “The translation initiation factor eIF-4B contains an RNA-binding region that is distinct and independent from its ribonucleoprotein consensus sequence," Molecular and Cellular Biology, vol. 14, no. 4, pp. 2307-2316, 1994.

[15] W. C. Merrick, "Eukaryotic protein synthesis: an in vitro analysis," Biochimie, vol. 76, no. 9, pp. 822-830, 1994.

[16] C. Wei, M. L. Balasta, J. Ren, and D. J. Goss, "Wheat germ poly(A) binding protein enhances the binding affinity of eukaryotic initiation factor $4 \mathrm{~F}$ and (iso) $4 \mathrm{~F}$ for cap analogues," Biochemistry, vol. 37, no. 7, pp. 1910-1916, 1998.

[17] X. Bi, J. Ren, and D. J. Goss, "Wheat germ translation initiation factor eIF4B affects eIF4A and eIFiso4F helicase activity by increasing the ATP binding affinity of eIF4A," Biochemistry, vol. 39, no. 19, pp. 5758-5765, 2000.

[18] D. R. Gallie, W. J. Lucas, and V. Walbot, "Visualizing mRNA expression in plant protoplasts: factors influencing efficient mRNA uptake and translation," The Plant Cell, vol. 1, no. 3, pp. 301-311, 1989.

[19] D. R. Gallie, “The cap and poly(A) tail function synergistically to regulate mRNA translational efficiency," Genes and Development, vol. 5, no. 11, pp. 2108-2116, 1991.

[20] D. R. Gallie and V. Walbot, "RNA pseudoknot domain of tobacco mosaic virus can functionally substitute for a poly(A) tail in plant an animal cells," Genes and Development, vol. 4, no. 7, pp. 1149-1157, 1990. 
[21] V. Leathers, R. Tanguay, M. Kobayashi, and D. R. Gallie, "A phylogenetically conserved sequence within viral $3 /$ untranslated RNA pseudoknots regulates translation," Molecular and Cellular Biology, vol. 13, no. 9, pp. 5331-5347, 1993.

[22] D. R. Gallie and M. Kobayashi, “The role of the 3 '-untranslated region of non-polyadenylated plant viral mRNAs in regulating translational efficiency," Gene, vol. 142, no. 2, pp. 159-165, 1994.

[23] D. R. Gallie, R. L. Tanguay, and V. Leathers, "The tobacco etch viral $5^{\prime}$ leader and poly(A) tail are functionally synergistic regulators of translation," Gene, vol. 165, no. 2, pp. 233-238, 1995.

[24] D. R. Gallie, N. J. Lewis, and W. F. Marzluff, "The histone 3/-terminal stem-loop is necessary for translation in Chinese hamster ovary cells," Nucleic Acids Research, vol. 24, no. 10, pp. 1954-1962, 1996.

[25] J. Ling, S. J. Morley, V. M. Pain, W. F. Marzluff, and D. R. Gallie, "The histone 3/-terminal stem-loop-binding protein enhances translation through a functional and physical interaction with eukaryotic initiation factor 4G (eIF4G) and eIF3," Molecular and Cellular Biology, vol. 22, no. 22, pp. 7853-7867, 2002.

[26] I. M. Krab, C. Caldwell, D. R. Gallie, and J. F. Bol, “Coat protein enhances translational efficiency of Alfalfa mosaic virus RNAs and interacts with the eIF4G component of initiation factor eIF4F," Journal of General Virology, vol. 86, no. 6, pp. 1841-1849, 2005.

[27] H. Le, R. L. Tanguay, M. L. Balasta et al., "Translation initiation factors eIF-iso4G and eIF-4B interact with the poly(A)-binding protein and increase its RNA binding activity," Journal of Biological Chemistry, vol. 272, no. 26, pp. 16247-16255, 1997.

[28] S. Z. Tarun Jr. and A. B. Sachs, "Association of the yeast poly(A) tail binding protein with translation initiation factor eIF-4G," EMBO Journal, vol. 15, no. 24, pp. 7168-7177, 1996.

[29] H. Imataka, A. Gradi, and N. Sonenberg, "A newly identified Nterminal amino acid sequence of human eIF4G binds poly(A)binding protein and functions in poly(A)-dependent translation," The EMBO Journal, vol. 17, no. 24, pp. 7480-7489, 1998.

[30] M. Piron, P. Vende, J. Cohen, and D. Poncet, "Rotavirus RNAbinding protein NSP3 interacts with eIF4GI and evicts the poly(A) binding protein from eIF4F," EMBO Journal, vol. 17, no. 19, pp. 5811-5821, 1998.

[31] C. S. Fraser, V. M. Pain, and S. J. Morley, "The association of initiation factor $4 \mathrm{~F}$ with poly(A)-binding protein is enhanced in serum-stimulated Xenopus kidney cells," The Journal of Biological Chemistry, vol. 274, no. 1, pp. 196-204, 1999.

[32] M. Bushell, W. Wood, G. Carpenter, V. M. Pain, S. J. Morley, and M. J. Clemens, "Disruption of the interaction of mammalian protein synthesis initiation factor $4 \mathrm{~B}$ with the poly(A) binding protein by caspase - and viral protease-mediated cleavages," The Journal of Biological Chemistry, vol. 276, no. 26, pp. 2392223928, 2001.

[33] L. Bellsolell, P. F. Cho-Park, F. Poulin, N. Sonenberg, and S. K. Burley, "Two structurally atypical HEAT domains in the Cterminal portion of human eIF4G support binding to eIF4A and Mnk1," Structure, vol. 14, no. 5, pp. 913-923, 2006.

[34] J. Marcotrigiano, I. B. Lomakin, N. Sonenberg, T. V. Pestova, C. U. T. Hellen, and S. K. Burley, "A conserved HEAT domain within eIF4G directs assembly of the translation initiation machinery," Molecular Cell, vol. 7, no. 1, pp. 193-203, 2001.

[35] S. Cheng and D. R. Gallie, "Competitive and noncompetitive binding of eIF4B, eIF4A, and the poly(A) binding protein to wheat translation initiation factor eIFiso4G," Biochemistry, vol. 49, no. 38, pp. 8251-8265, 2010.
[36] S. Cheng and D. R. Gallie, "Eukaryotic initiation factor 4B and the poly(A)-binding protein bind eIF4G competitively," Translation, vol. 1, no. 1, Article ID e24038, 13 pages, 2013.

[37] C. Goyer, M. Altmann, H. S. Lee et al., "TIF4631 and TIF4632: two yeast genes encoding the high-molecular-weight subunits of the cap-binding protein complex (eukaryotic initiation factor $4 \mathrm{~F}$ ) contain an RNA recognition motif-like sequence and carry out an essential function," Molecular and Cellular Biology, vol. 13, no. 8, pp. 4860-4874, 1993.

[38] B. J. Lamphear, R. Kirchweger, T. Skern, and R. E. Rhoads, "Mapping of functional domains in eukaryotic protein synthesis initiation factor $4 \mathrm{G}$ (eIF4G) with picornaviral proteases. Implications for Cap-dependent and Cap-independent translational initiation," Journal of Biological Chemistry, vol. 270, no. 37, pp. 21975-21983, 1995.

[39] H. Imataka and N. Sonenberg, "Human eukaryotic translation initiation factor $4 \mathrm{G}$ (eIF4G) possesses two separate and independent binding sites for eIF4A," Molecular and Cellular Biology, vol. 17, no. 12, pp. 6940-6947, 1997.

[40] A. Marintchev and G. Wagner, "Translation initiation: structures, mechanisms and evolution," Quarterly Reviews of Biophysics, vol. 37, no. 3-4, pp. 197-284, 2004.

[41] D. R. Gallie and K. S. Browning, "eIF4G functionally differs from eIFiso4G in promoting internal initiation, capindependent translation, and translation of structured mRNAs," The Journal of Biological Chemistry, vol. 276, no. 40, pp. 3695136960, 2001.

[42] A. K. Lefebvre, N. L. Korneeva, M. Trutschl et al., "Translation initiation factor eIF4G-1 binds to eIF3 through the eIF3e subunit," Journal of Biological Chemistry, vol. 281, no. 32, pp. 22917-22932, 2006.

[43] N. Villa, A. Do, J. W. Hershey, and C. S. Fraser, "Human eukaryotic initiation factor $4 \mathrm{G}$ (eIF4G) protein binds to eIF3c, -d, and -e to promote mRNA recruitment to the ribosome," The Journal of Biological Chemistry, vol. 288, no. 46, pp. 3293232940, 2013.

[44] N. L. Korneeva, B. J. Lamphear, F. L. C. Hennigan, and R. E. Rhoads, "Mutually cooperative binding of eukaryotic translation initiation factor (eIF) 3 and eIF4A to human eIF4G1," The Journal of Biological Chemistry, vol. 275, no. 52, pp. 41369-41376, 2000.

[45] H. He, T. von der Haar, C. R. Singh et al., "The yeast eukaryotic initiation factor 4G (eIF4G) HEAT domain interacts with eIF1 and eIF5 and is involved in stringent AUG selection," Molecular and Cellular Biology, vol. 23, no. 15, pp. 5431-5445, 2003.

[46] C. R. Singh, R. Watanabe, W. Chowdhury et al., "Sequential eukaryotic translation initiation factor 5 (eIF5) binding to the charged disordered segments of eIF4G and eIF $2 \beta$ stabilizes the $48 \mathrm{~S}$ preinitiation complex and promotes its shift to the initiation mode," Molecular and Cellular Biology, vol. 32, no. 19, pp. 39783989, 2012.

[47] Y. Yamamoto, C. R. Singh, A. Marintchev et al., "The eukaryotic initiation factor (eIF) 5 HEAT domain mediates multifactor assembly and scanning with distinct to interacts to eIF1, eIF2, eIF3, and eIF4G," Proceedings of the National Academy of Sciences of the United States of America, vol. 102, no. 45, pp. 16164-16169, 2005.

[48] A. Gradi, H. Imataka, Y. V. Svitkin et al., "A novel functional human eukaryotic translation initiation factor 4G," Molecular and Cellular Biology, vol. 18, no. 1, pp. 334-342, 1998.

[49] K. S. Browning, "The plant translational apparatus," Plant Molecular Biology, vol. 32, no. 1-2, pp. 107-144, 1996. 
[50] I. B. Lomakin, C. U. T. Hellen, and T. V. Pestova, "Physical association of eukaryotic initiation factor $4 \mathrm{G}$ (EIF4G) with EIF4A strongly enhances binding of EIF4G to the internal ribosomal entry site of encephalomyocarditis virus and is required for internal initiation of translation," Molecular and Cellular Biology, vol. 20, no. 16, pp. 6019-6029, 2000.

[51] P. Schütz, M. Bumann, A. E. Oberholzer et al., "Crystal structure of the yeast eIF4A-eIF4G complex: an RNA-helicase controlled by protein-protein interactions," Proceedings of the National Academy of Sciences of the United States of America, vol. 105, no. 28, pp. 9564-9569, 2008.

[52] H. S. Yang, M. H. Cho, H. Zakowicz, G. Hegamyer, N. Sonenberg, and N. H. Colburn, "A novel function of the MA3 domains in transformation and translation suppressor Pdcd 4 is essential for its binding to eukaryotic translation initiation factor 4A," Molecular and Cellular Biology, vol. 24, no. 9, pp. 3894-3906, 2004.

[53] H. Le, K. S. Browning, and D. R. Gallie, "The phosphorylation state of poly(A)-binding protein specifies its binding to poly(A) RNA and its interaction with eukaryotic initiation factor (eIF) 4F, eIFiso4F, and eIF4B," The Journal of Biological Chemistry, vol. 275, no. 23, pp. 17452-17462, 2000.

[54] A. Z. Andreou and D. Klostermeier, "eIF4B and eIF4G jointly stimulate eIF4A ATPase and unwinding activities by modulation of the eIF4A conformational cycle," Journal of Molecular Biology, vol. 426, pp. 51-61, 2014.

[55] S. Lax, W. Fritz, K. Browning, and J. Ravel, "Isolation and characterization of factors from wheat germ that exhibit eukaryotic initiation factor $4 \mathrm{~B}$ activity and overcome 7-methylguanosine 5'-triphosphate inhibition of polypeptide synthesis," Proceedings of the National Academy of Sciences of the United States of America, vol. 82, no. 2, pp. 330-333, 1985.

[56] S. R. Lax, K. S. Browning, D. M. Maia, and J. M. Ravel, "ATPase activities of wheat germ initiation factors $4 \mathrm{~A}, 4 \mathrm{~B}$, and $4 \mathrm{~F}$," Journal of Biological Chemistry, vol. 261, no. 33, pp. 15632-15636, 1986.

[57] K. S. Browning, S. R. Lax, and J. M. Ravel, "Identification of two messenger RNA cap binding proteins in wheat germ. Evidence that the $28-\mathrm{kD}$ a subunit of eIF-4B and the $26-\mathrm{kDa}$ subunit of eIF-4F are antigenically distinct polypeptides," The Journal of Biological Chemistry, vol. 262, no. 23, pp. 11228-11232, 1987.

[58] K. S. Browning, L. Fletcher, S. R. Lax, and J. M. Ravel, "Evidence that the $59-\mathrm{kDa}$ protein synthesis initiation factor from wheat germ is functionally similar to the $80-\mathrm{kDa}$ initiation factor $4 \mathrm{~B}$ from mammalian cells," Journal of Biological Chemistry, vol. 264, no. 15, pp. 8491-8494, 1989.

[59] G. W. Rogers Jr., N. J. Richter, and W. C. Merrick, "Biochemical and kinetic characterization of the RNA helicase activity of eukaryotic initiation factor 4A," Journal of Biological Chemistry, vol. 274, no. 18, pp. 12236-12244, 1999.

[60] G. W. Rogers Jr., N. J. Richter, W. F. Lima, and W. C. Merrick, "Modulation of the Helicase Activity of eIF4A by eIF4B, eIF4H, and eIF4F," The Journal of Biological Chemistry, vol. 276, no. 33, pp. 30914-30922, 2001.

[61] H. Trachsel, B. Erni, M. H. Schreier, and T. Staehelin, "Initiation of mammalian protein synthesis. II. The assembly of the initiation complex with purified initiation factors," Journal of Molecular Biology, vol. 116, no. 4, pp. 755-767, 1977.

[62] R. Benne and J. W. B. Hershey, "The mechanism of action of protein synthesis initiation factors from rabbit reticulocytes," Journal of Biological Chemistry, vol. 253, no. 9, pp. 3078-3087, 1978.
[63] M. Altmann, B. Wittmer, N. Methot, N. Sonenberg, and H. Trachsel, "The Saccharomyces cerevisiae translation initiation factor Tif3 and its mammalian homologue, eIF-4B, have RNA annealing activity," The EMBO Journal, vol. 14, no. 15, pp. 38203827, 1995.

[64] T. V. Pestova, I. N. Shatsky, and C. U. T. Hellen, "Functional dissection of eukaryotic initiation factor $4 \mathrm{~F}$ : the $4 \mathrm{~A}$ subunit and the central domain of the $4 \mathrm{G}$ subunit are sufficient to mediate internal entry of $43 \mathrm{~S}$ preinitiation complexes," Molecular and Cellular Biology, vol. 16, no. 12, pp. 6859-6869, 1996.

[65] S. Morino, H. Imataka, Y. V. Svitkin, T. V. Pestova, and N. Sonenberg, "Eukaryotic translation initiation factor $4 \mathrm{E}$ (eIF4E) binding site and the middle one-third of eIF4GI constitute the core domain for cap-dependent translation, and the C-terminal one-third functions as a modulatory region," Molecular and Cellular Biology, vol. 20, no. 2, pp. 468-477, 2000.

[66] S. Cheng and D. R. Gallie, "Wheat eukaryotic initiation factor $4 \mathrm{~B}$ organizes assembly of RNA and eIFiso4G, eIF4A, and poly(A)-binding protein," The Journal of Biological Chemistry, vol. 281, no. 34, pp. 24351-24364, 2006.

[67] F. Zhou, S. E. Walker, S. F. Mitchell, J. R. Lorsch, and A. G. Hinnebusch, "Identification and characterization of functionally critical, conserved motifs in the internal repeats and N-terminal domain of yeast translation initiation factor 4B (yeIF4B)," The Journal of Biological Chemistry, vol. 289, no. 3, pp. 1704-1722, 2014.

[68] A. B. Sachs, R. W. Davis, and R. D. Kornberg, "A single domain of yeast poly(A)-binding protein is necessary and sufficient for RNA binding and cell viability," Molecular and Cellular Biology, vol. 7, no. 9, pp. 3268-3276, 1987.

[69] U. Kühn and T. Pieler, "XenopusPoly(A) binding protein: functional domains in RNA binding and protein-protein interaction," Journal of Molecular Biology, vol. 256, no. 1, pp. 20 30, 1996.

[70] S. H. Kessler and A. B. Sachs, "RNA recognition motif 2 of yeast Pablp is required for its functional interaction with eukaryotic translation initiation factor 4G," Molecular and Cellular Biology, vol. 18, no. 1, pp. 51-57, 1998.

[71] L. J. Otero, M. P. Ashe, and A. B. Sachs, "The yeast poly(A)binding protein Pablp stimulates in vitro poly(A)-dependent and cap-dependent translation by distinct mechanisms," EMBO Journal, vol. 18, no. 11, pp. 3153-3163, 1999.

[72] S. Cheng and D. R. Gallie, "eIF4G, eIFiso4G, and eIF4B bind the poly(A)-binding protein through overlapping sites within the RNA recognition motif domains," Journal of Biological Chemistry, vol. 282, no. 35, pp. 25247-25258, 2007.

[73] K. Khaleghpour, Y. V. Svitkin, A. W. Craig et al., "Translational repression by a novel partner of human poly(A) binding protein, Paip2," Molecular Cell, vol. 7, no. 1, pp. 205-216, 2001.

[74] G. Roy, G. de Crescenzo, K. Khaleghpour, A. Kahvejian, M. O'Connor-McCourt, and N. Sonenberg, "Paipl interacts with poly(A) binding protein through two independent binding motifs," Molecular and Cellular Biology, vol. 22, no. 11, pp. 37693782, 2002.

[75] N. Uchida, S. Hoshino, H. Imataka, N. Sonenberg, and T. Katada, "A novel role of the mammalian GSPT/eRF3 associating with poly(A)-binding protein in cap/poly(A)-dependent translation," Journal of Biological Chemistry, vol. 277, no. 52, pp. 50286-50292, 2002.

[76] N. Hosoda, T. Kobayashi, N. Uchida et al., “Translation termination factor eRF3 mediates mRNA decay through the regulation 
of deadenylation," Journal of Biological Chemistry, vol. 278, no. 40, pp. 38287-38291, 2003.

[77] M. Albrecht and T. Lengauer, "Survey on the PABC recognition motif PAM2," Biochemical and Biophysical Research Communications, vol. 316, no. 1, pp. 129-138, 2004.

[78] X. Wang and R. Grumet, "Identification and characterization of proteins that interact with the carboxy terminus of poly(A)binding protein and inhibit translation in vitro," Plant Molecular Biology, vol. 54, no. 1, pp. 85-98, 2004.

[79] J. Bravo, L. Aguilar-Henonin, G. Olmedo, and P. Guzmán, "Four distinct classes of proteins as interaction partners of the PABC domain of Arabidopsis thaliana poly(A)-binding proteins," Molecular Genetics and Genomics, vol. 272, no. 6, pp. 651-665, 2005.

[80] N. Siddiqui, M. J. Osborne, D. R. Gallie, and K. Gehring, "Solution structure of the PABC domain from wheat poly (A)binding protein: an insight into RNA metabolic and translational control in plants," Biochemistry, vol. 46, no. 14, pp. 42214231, 2007.

[81] S. Cheng, S. Sultana, D. J. Goss, and D. R. Gallie, "Translation initiation factor 4B homodimerization, RNA binding, and interaction with poly(A)-binding protein are enhanced by zinc," Journal of Biological Chemistry, vol. 283, no. 52, pp. 36140-36153, 2008.

[82] Y. M. Michel, D. Poncet, M. Piron, K. M. Kean, and A. M. Borman, "Cap-poly(A) synergy in mammalian cell-free extracts. Investigation of the requirements for poly(A)-mediated stimulation of translation initiation," The Journal of Biological Chemistry, vol. 275, no. 41, pp. 32268-32276, 2000.

[83] T. von der Haar, P. D. Ball, and J. E. G. McCarthy, "Stabilization of eukaryotic initiation factor $4 \mathrm{E}$ binding to the mRNA 5/-cap by domains of eIF4G," The Journal of Biological Chemistry, vol. 275, no. 39, pp. 30551-30555, 2000.

[84] A. Kahvejian, Y. V. Svitkin, R. Sukarieh, M. M'Boutchou, and N. Sonenberg, "Mammalian poly(A)-binding protein is a eukaryotic translation initiation factor, which acts via multiple mechanisms," Genes and Development, vol. 19, no. 1, pp. 104-113, 2005.

[85] T. M. Hinton, M. J. Coldwell, G. A. Carpenter, S. J. Morley, and V. M. Pain, "Functional analysis of individual binding activities of the scaffold protein eIF4G," The Journal of Biological Chemistry, vol. 282, no. 3, pp. 1695-1708, 2007.

[86] Y. Luo and D. J. Goss, "Homeostasis in mRNA initiation: wheat germ poly(A)-binding protein lowers the activation energy barrier to initiation complex formation," The Journal of Biological Chemistry, vol. 276, no. 46, pp. 43083-43086, 2001.

[87] M. A. Khan and D. J. Goss, “Translation initiation factor (eIF) $4 \mathrm{~B}$ affects the rates of binding of the mRNA $\mathrm{m} 7 \mathrm{G}$ cap analogue to wheat germ eIFiso4F and eIFiso4F.PABP," Biochemistry, vol. 44, no. 11, pp. 4510-4516, 2005.

[88] N. Amrani, S. Ghosh, D. A. Mangus, and A. Jacobson, "Translation factors promote the formation of two states of the closedloop mRNP," Nature, vol. 453, no. 7199, pp. 1276-1280, 2008.

[89] Y. V. Svitkin, V. M. Evdokimova, A. Brasey et al., "General RNAbinding proteins have a function in poly(A)-binding proteindependent translation," EMBO Journal, vol. 28, no. 1, pp. 58-68, 2009.

[90] S. E. Wells, P. E. Hillner, R. D. Vale, and A. B. Sachs, "Circularization of mRNA by eukaryotic translation initiation factors," Molecular Cell, vol. 2, no. 1, pp. 135-140, 1998.
[91] N. Iizuka, L. Najita, A. Franzusoff, and P. Sarnow, "Capdependent and cap-independent translation by internal initiation of mRNAs in cell extracts prepared from Saccharomyces cerevisiae," Molecular and Cellular Biology, vol. 14, no. 11, pp. 7322-7330, 1994.

[92] S. Z. Tarun Jr. and A. B. Sachs, "A common function for mRNA 5 ' and 3' ends in translation initiation in yeast," Genes and Development, vol. 9, no. 23, pp. 2997-3007, 1995.

[93] D. R. Gallie, "The role of the initiation surveillance complex in promoting efficient protein synthesis," Biochemical Society Transactions, vol. 32, no. 4, pp. 585-588, 2004.

[94] B. W. Baer and R. D. Kornberg, "Repeating structure of cytoplasmic poly(A)-ribonucleoprotein.", Proceedings of the National Academy of Sciences of the United States of America, vol. 77, no. 4, pp. 1890-1892, 1980.

[95] N. Méthot, M. S. Song, and N. Sonenberg, "A region rich in aspartic acid, arginine, tyrosine, and glycine (DRYG) mediates eukaryotic initiation factor 4B (eIF4B) self-association and interaction with eIF3," Molecular and Cellular Biology, vol. 16, no. 10, pp. 5328-5334, 1996.

[96] H. Park, K. S. Browning, T. Hohn, and L. A. Ryabova, "Eucaryotic initiation factor $4 \mathrm{~B}$ controls eIF3-mediated ribosomal entry of viral reinitiation factor," EMBO Journal, vol. 23, no. 6, pp. 1381-1391, 2004.

[97] J. Drawbridge, J. L. Grainger, and M. M. Winkler, "Identification and characterization of the poly(A)-binding proteins from the sea urchin: a quantitative analysis," Molecular and Cellular Biology, vol. 10, no. 8, pp. 3994-4006, 1990.

[98] D. R. Gallie, H. Le, C. Caldwell, R. L. Tanguay, N. X. Hoang, and K. S. Browning, "The phosphorylation state of translation initiation factors is regulated developmentally and following heat shock in wheat," The Journal of Biological Chemistry, vol. 272, no. 2, pp. 1046-1053, 1997.

[99] D. C. Schwartz and R. Parker, "Interaction of mRNA translation and mRNA degradation in Saccharomyces cerevisiae," in Translational Control of Gene Expression, N. Sonenberg, J. W. B. Hershey, and M. B. Mathews, Eds., pp. 807-825, Cold Spring Harbor Press, New York, NY, USA, 2000.

[100] H. Le, S. Chang, R. L. Tanguay, and D. R. Gallie, "The wheat poly(A)-binding protein functionally complements pab1 in yeast," European Journal of Biochemistry, vol. 243, no. 1-2, pp. 350-357, 1997.

[101] R. Duncan and J. W. B. Hershey, "Regulation of initiation factors during translational repression caused by serum depletion. Covalent modification," The Journal of Biological Chemistry, vol. 260, no. 9, pp. 5493-5497, 1985.

[102] R. F. Duncan and J. W. B. Hershey, "Protein synthesis and protein phosphorylation during heat stress, recovery, and adaptation," Journal of Cell Biology, vol. 109, no. 4, pp. 1467-1481, 1989.

[103] J. M. Manzella, W. Rychlik, R. E. Rhoads, J. W. B. Hershey, and P. J. Blackshear, "Insulin induction of ornithine decarboxylase: importance of mRNA secondary structure and phosphorylation of eucaryotic initiation factors eIF-4B and eIF-4E," The Journal of Biological Chemistry, vol. 266, no. 4, pp. 2383-2389, 1991.

[104] M. Wakiyama, H. Imataka, and N. Sonenberg, "Interaction of elF4G with poly(A)-binding protein stimulates translation and is critical for Xenopus oocyte maturation," Current Biology, vol. 10, no. 18, pp. 1147-1150, 2000. 
[105] I. K. Ali, L. McKendrick, S. J. Morley, and R. J. Jackson, "Truncated initiation factor eIF4G lacking an eIF4E binding site can support capped mRNA translation," EMBO Journal, vol. 20, no. 15, pp. 4233-4242, 2001.

[106] D. R. Gallie, “The 5/-leader of tobacco mosaic virus promotes translation through enhanced recruitment of elF4F”, Nucleic Acids Research, vol. 30, no. 15, pp. 3401-3411, 2002. 

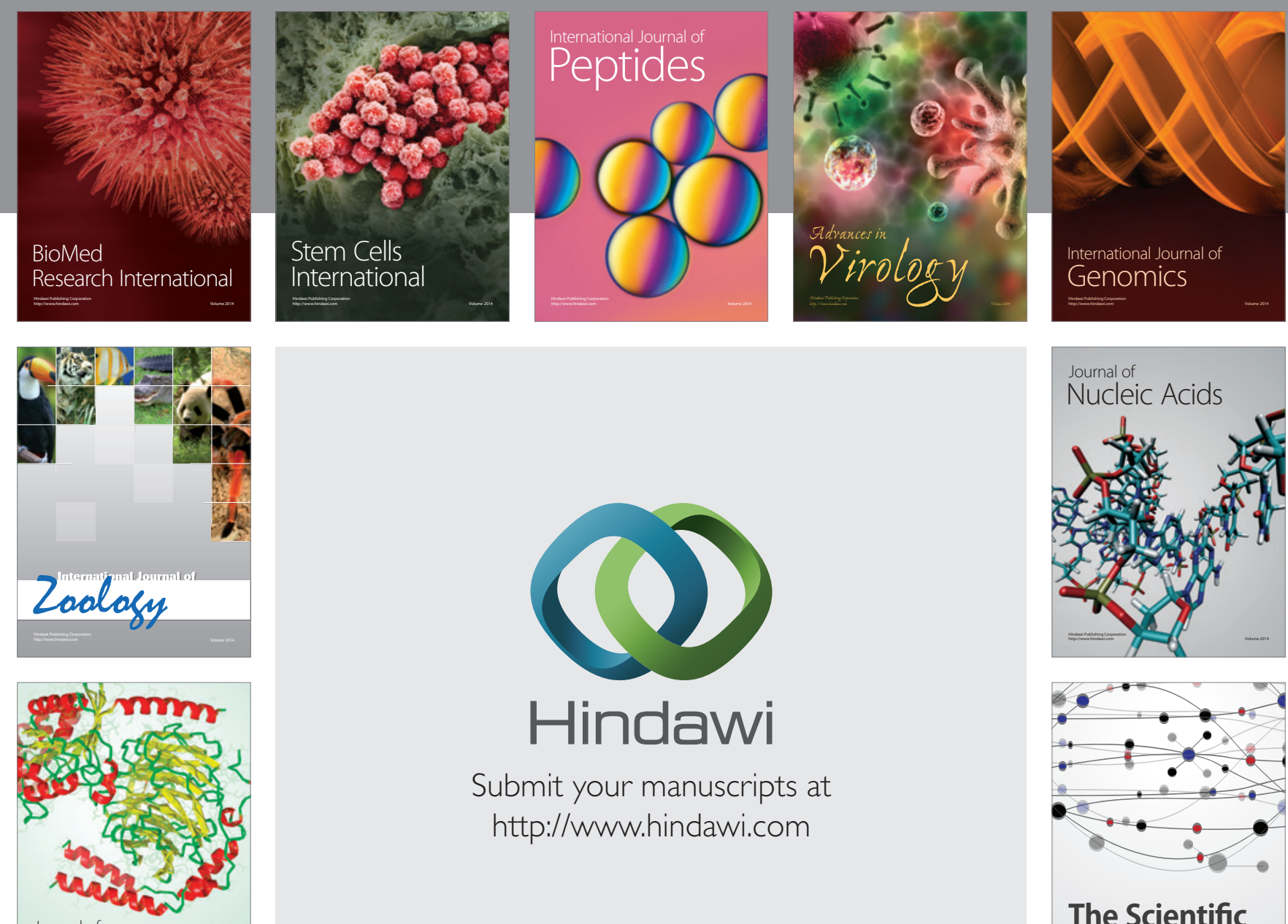

Submit your manuscripts at

http://www.hindawi.com

Journal of
Signal Transduction
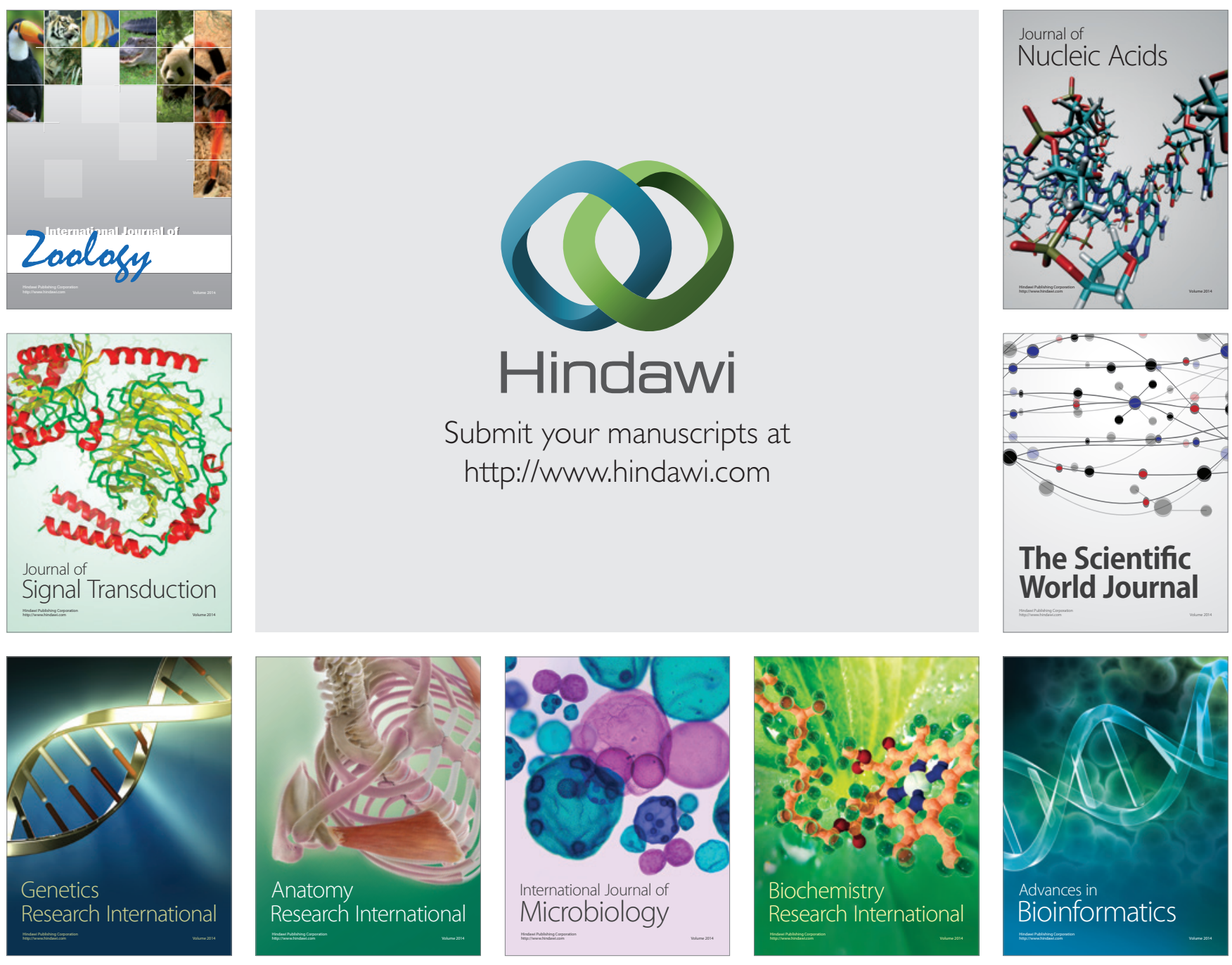

The Scientific World Journal
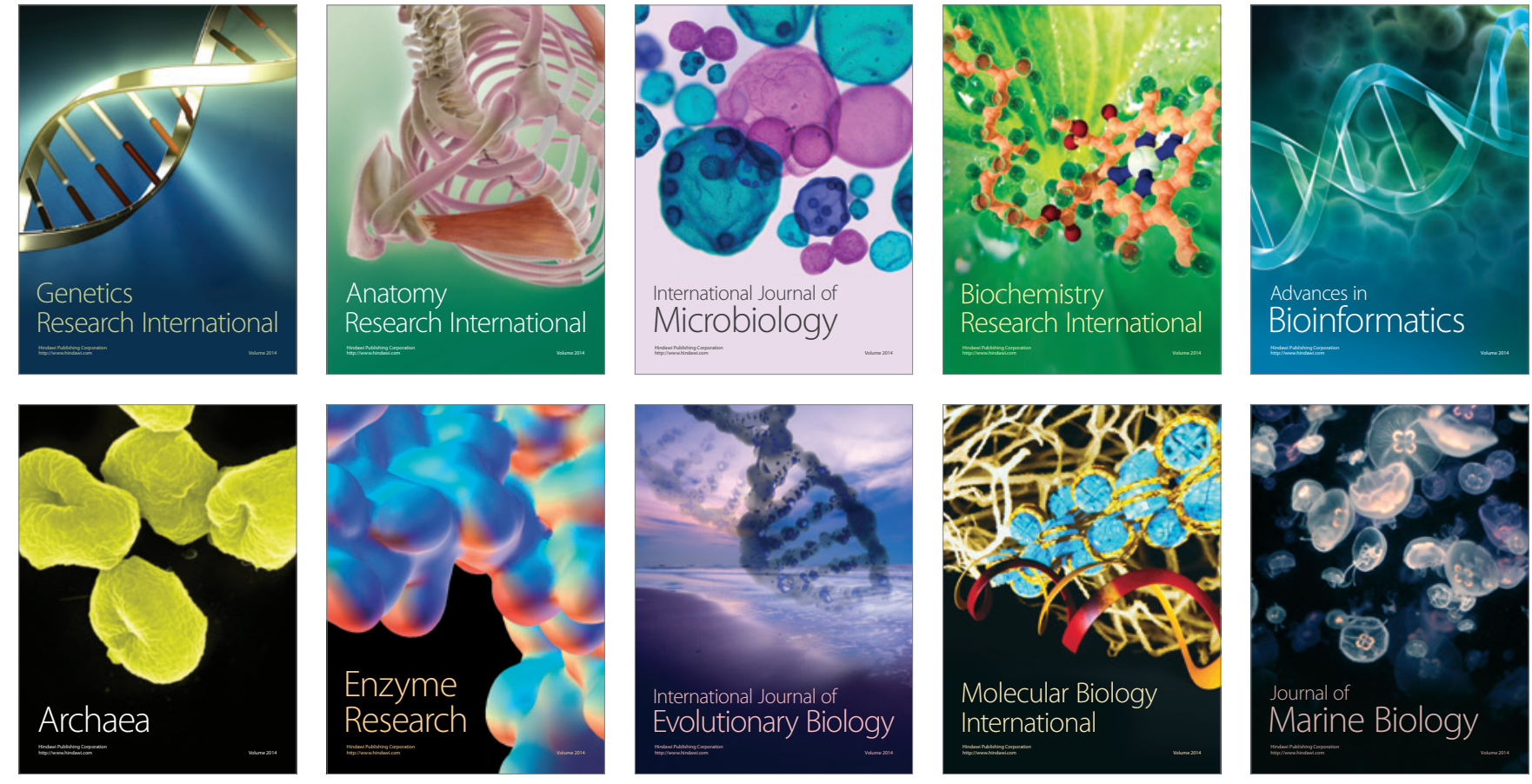